\title{
LONG-TERM POPULATION TRENDS OF SEABIRDS ON THE SWAIN REEFS, GREAT BARRIER REEF
}

\section{H. Heatwole ${ }^{1}$, P. O’Neill ${ }^{2}$, M. Jones ${ }^{3}$ and M. Preker ${ }^{4}$}

${ }^{1}$ Department of Zoology, North Carolina State University, Raleigh, NC, 27695-7617, USA.

${ }^{2}$ Queensland Department of Environment, PO Box 3130, Rockhampton Shopping Fair, Qld. 4701, Australia.

${ }^{3}$ Department of Zoology, University of Tasmania, Hobart, Tas. 7005, Australia.

${ }^{4} 321$ Pullenvale Road, Pullenvale, Qld. 4069, Australia.

A report funded by the CRC Reef Research Centre.

The CRC Reef Research Centre was established under the Australian Government's Cooperative Research Centres Program.

The Centre, established in 1993, undertakes an integral program of applied research and development, training and education, aimed at increasing opportunities for ecologically sustainable development of the Great Barrier Reef and providing an improved scientific basis for Reef management and regulatory decision making.

CRC Reef Research Centre

c/- James Cook University

TOWNSVILLE QLD 4811

Phone: (077) 814976

Fax: (077) 814099 
Email: crc.reef@jcu.edu.au 
? Cooperative Research Centre for Ecologically Sustainable Development of the Great Barrier Reef

National Library of Australia Cataloguing-inPublication entry

Heatwole, H., O’Neill, P., Jones, M., \& Preker, M. Long-term population trends of seabirds on the Swain Reefs, Great Barrier Reef

Bibliography.

Includes index.

ISBN 1876054115.

1. Bird populations - Queensland - Swain Reefs Region. 2. Sea birds - Queensland - Swain Reefs Region. I. Heatwole, Harold. II. Cooperative Research Centre for Ecologically Sustainable Development of the Great Barrier Reef (Australia). III. Title. (Series: CRC Reef Research technical report; 12).

598.29943

This publication should be cited as:

Heatwole, H., O’Neill, P., Jones, M., \& Preker, M. (1996)

Long-term population trends of seabirds on the Swain Reefs, Great Barrier Reef

Technical Report No. 12

Townsville; CRC Reef Research Centre, 50 pp.

This work is copyright. The Copyright Act 1968 permits fair dealing for study, research, news reporting, criticism or review. Selected passages, tables or diagrams may be reproduced for such purposes provided acknowledgement of the source is included. Major extracts of the entire document may not be reproduced by any process without written permission of the Director, CRC Reef Research Centre.

Published by the Cooperative Research Centre for Ecologically Sustainable Development of the Great Barrier Reef.

Further copies may be obtained from CRC Reef Research Centre, c/- James Cook University Post Office, Townsville, QLD 4811.

Printed by James Cook University of North Queensland. 


\section{TABLE OF CONTENTS}

\section{SUMMARY}

2. INTRODUCTION

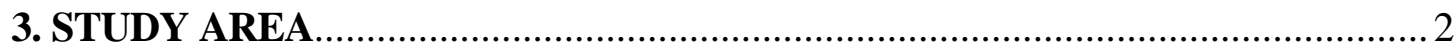

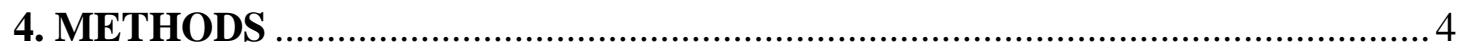

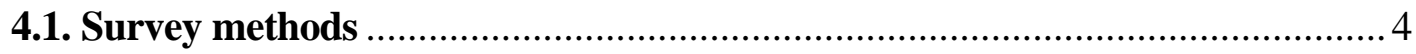

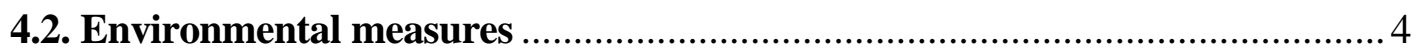

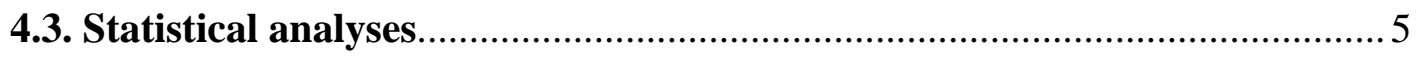

5. RESULTS

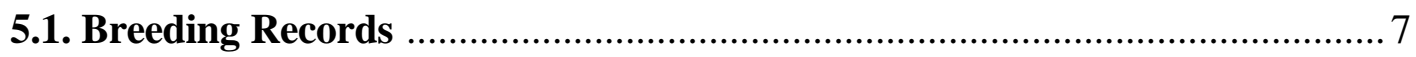

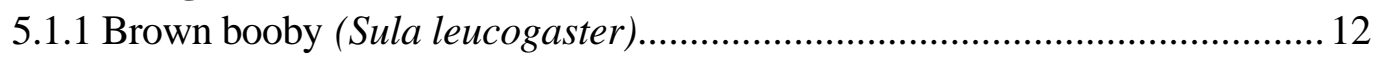

5.1.2 Masked booby (Sula dactylatra) .............................................................. 16

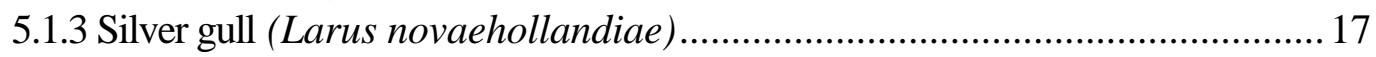

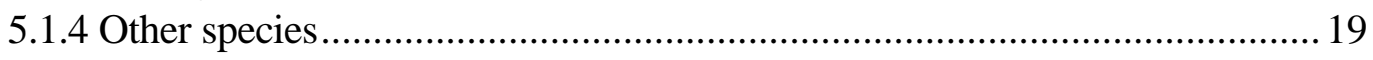

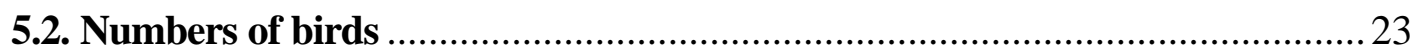

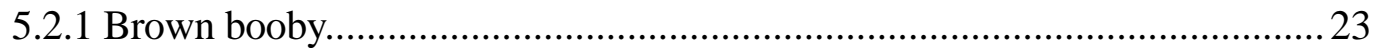

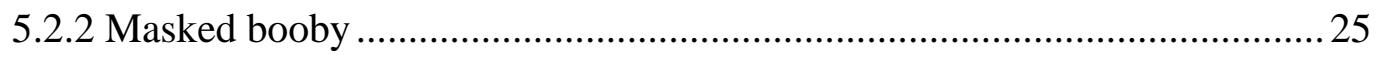

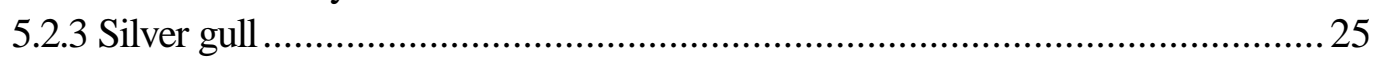

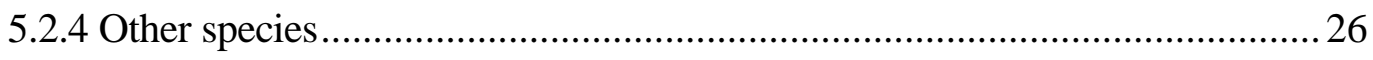

5.3. Weather conditions and patterns of change on islands ............................ 27

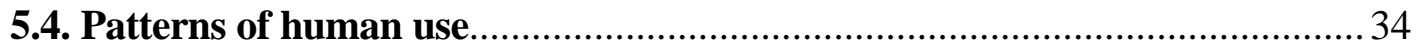

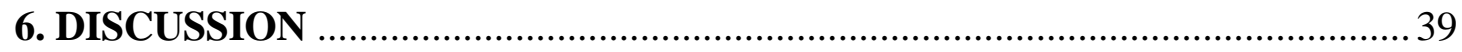

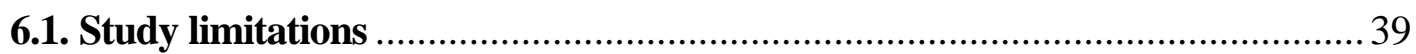

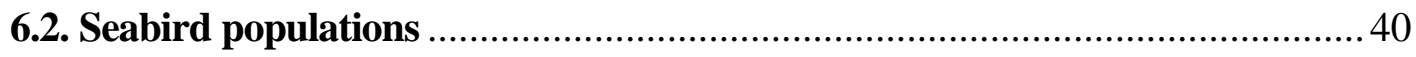

6.3. Management of seabird islands in the Swain Reefs ................................ 46

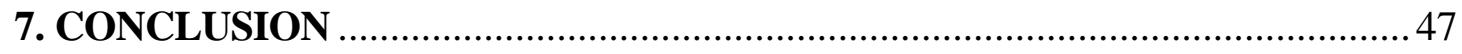

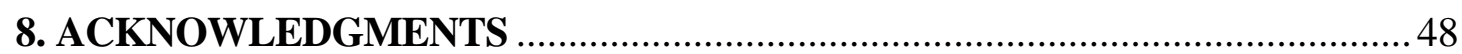


9. REFERENCES 


\section{LIST OF FIGURES}

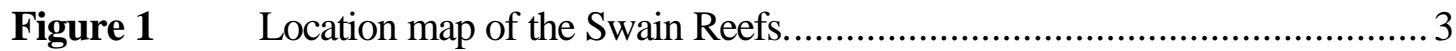

Figure 2 Numbers of brown boobies recorded between 1980 and 1995 in the Swain

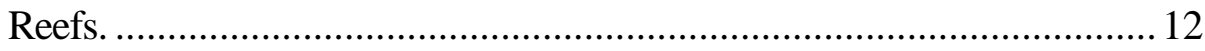

Figure 3 Numbers of masked booby nests recorded between 1980 and 1995 on seven cays in the Swain Reefs.......................................................... 16

Figure 4 Numbers of brown booby adults recorded between 1980 and 1995 on seven cays in the Swain Reefs.

Figure 5 Numbers of masked booby adults recorded between 1980 and 1995 on seven cays in the Swain Reefs.

Figure 6 Numbers of silver gull adults recorded between 1980 and 1995 on seven cays in the Swain Reefs. 25

Figure 7 Overlays showing temporal changes to shape and size, relative location and boundary of vegetation for three currently vegetated cays in the Swain Reefs.

Figure 8 Overlays showing temporal changes to shape and size, relative location and boundary of vegetation for three currently unvegetated cays in the Swain Reefs. 32

Figure 9a Monthly variation of Southern Oscillation Index from 1975 to 1995. 33

Figure 9b Monthly sea surface temperatures for the Great Barrier Reef latitude band

?? S to $22 \mathrm{~S}$ for the years 1975 to 1995 33

Figure 9c Mean monthly air temperatures recorded at Gannet Cay at $1 \mathrm{pm}$ daily from 1975 to 1995. 34

Figure 9d Mean monthly wind speeds recorded at Gannet Cay at $1 \mathrm{pm}$ daily from 1975 to 1995. 34

Figure 10 Graphs of numbers of primary fishing vessels, secondary fishing vessels and charter vessels recorded by aerial surveillance in plots $F$ and $G$ in the Swain Reefs, from 1988 to 1994. 


\section{LIST OF TABLES}

Table 1 Status and location of bird species recorded in the Swain Reefs. ..................

Table 2 Estimated numbers of breeding pairs of seabirds present during their respective principal breeding seasons on Swain Reef cays, in the periods Pre 1985, 1985-1989 and 1990-1994. 10

Table 3 Statistical difference from zero for slopes of regression lines fitted to summer and winter counts of adults and nests of brown and masked boobies, and adults of silver gulls.

Table 4 Slopes of regression lines fitted to counts of adults and nests of brown and masked boobies and adults of silver gulls for each cay.....

Table 5 Comparison (chi-squared tests) of means of summer and winter counts of adults and nests of brown and masked boobies, and adults of silver gulls.

Table 6 Minimum area of cay above High Water mark recorded per nest site between 1985 and 1995, for the primary brown and masked booby breeding cays in the Swain Reefs.

Table 7 Cyclones passing within 100 kilometres of the Swain Reefs in the period 1971 to 1995 . 27

Table 8 Total and vegetated areas of cays as measured from vertical aerial photographs flown in July 1964, November 1984, June 1992, and OctoberNovember 1993, and from ground measurements in July 1983, January and July 1984, and January 1985. 28

\section{LIST OF APPENDICES}

Appendix 1 Record of ornithological expeditions to the Swain Reef's cays .50 


\section{FOREWORD}

Seabirds are a significant fauna of the Great Barrier Reef. About 700000 pairs of 22 species are estimated to breed on the Reef islands, including significant proportions of many of the Australian breeding populations. However, life is becoming more problematic for seabirds on the Reef. In a number of places they now share their breeding islands with resort guests, campers and day visitors. Some, such as black noddies and wedge-tailed shearwaters appear to be coping and in places even increasing in numbers in the presence of people. However, the large majority are very sensitive to human disturbance and breeding numbers have declined on a number of islands, in some places resulting in abandonment of original breeding locations.

The scale of threats to seabirds on the Great Barrier Reef appears to be ever-expanding. Modern technology and mass transportation is providing for ever-increasing numbers of people to experience the wonders of the Reef. Although the changing Reef visitation scene may have adverse impacts on seabirds, the impacts may not be readily apparent. The main criterion for identifying an adverse impact that is underway is a decline in the number of birds present. Often this can only revealed through long-term studies of populations. Such is the nature of the Swains Reef seabird data analysed in this report commissioned by the CRC Reef Research Centre.

My first contact with Paul O'Neill was in 1992, when he spoke of a desire to analyse the wealth of data from the remote Swains Reef area which had been assiduously collected over the years by Professor Harold Heatwole and his co-workers. Although I have not met Professor Heatwole, time has proven that both he and Paul had the necessary commitment and dedication for the project to succeed. Under funding from CRC Reef, Paul spent six months in the United States of America working on the data with Professor Heatwole and his colleagues. The analyses combined with their excellent field knowledge of the Swains has enabled the value of the accumulating data to be realised.

All involved with the project should be commended. The results will be most useful in future planning and management of the seabirds in this special Reef area.

\section{Tony Stokes}

Threatened Species Program

Great Barrier Reef Marine Park Authority 


\section{SUMMARY}

The populations of seabirds inhabiting the Swain Reefs, Great Barrier Reef, were censused at six-monthly intervals from 1982 to 1994. Some cays are more important for seabird breeding than others, and an appropriate management strategy is outlined. Difficulties with censusing some species did not allow a reliable assessment of population trends, and these are reported in general terms only.

Data for three species, the masked booby (Sula dactylatra), the brown booby (Sula leucogaster) and the silver gull (Larus novaehollandiae) were analysed for trends in numbers of adults and numbers of nests counted. Significant declines in the numbers of adults and nests of the brown booby, and in the number of adult silver gulls, were detected during the period of the study.

Minimal human disturbance has occurred on the Swain Reefs cays compared with many other seabird breeding islands on the Great Barrier Reef. A range of natural and human factors were investigated for possible causes of the observed seabird declines, and human factors do not appear to have been influential. Reductions in available food, probably associated with elevated sea surface temperatures induced by El Niño, appears to be the most likely cause of the declines.

Trends in seabird populations observed in the Swain Reefs are useful baselines with which to compare populations from more disturbed areas. Such comparisons are necessary to separate impacts on seabird populations caused by human factors from those that occur naturally. 


\section{INTRODUCTION}

That the Swain Reefs were remote, uncharted and a serious navigation hazard protected them and their fauna from human disturbance until recent times. The first recorded visit, (noted by the naturalist J. Beete Jukes in his 1847 narrative of the surveying voyage of the H.M.S. Fly) was by Mr. Swain in the brig, Eliza, in 1798. H.M.S. Fly only briefly explored the edge of the reefs but an accompanying ship, H.M.S. Bramble, entered the complex. Although fishermen are known to have used the area during the past hundred years, the first biological expedition to the area was not until 1960 when an Honorary Associate of the Australian Museum (Mr. Len Thomas) made a short visit and discovered a number of uncharted cays (Gillet \& McNeill 1962). Hindwood (1961), McMichael (1963), Costello (1978), Limpus \& Lyon (1981) and Moverley (1985) all added to the knowledge of the avifauna of the area during brief visits.

Census methods used on many of the earliest expeditions are unknown, but some were less rigorous than others. Systematic investigation of these islands emerged only in the late 1970's with the launching of an integrated, interdisciplinary study emphasising the interactive roles of physical forces, soils, vegetation, birds and sea turtles in the Swain Reefs ecosystem. Papers on geomorphology (Flood and Heatwole 1986), vegetation dynamics (Heatwole 1987) and vegetation dispersal (Smith et al. 1990) have been published and a comprehensive account of soils and nutrient cycling is in preparation (J. Charley, pers. comm.). Heatwole (1991) presented a model of the dynamics of this ecosystem.

Concise information on cay morphology, ornithological history, and the status of breeding and non-breeding seabirds has previously been reported for individual cays in the Swain Reefs (Walker \& Jones 1986a, 1986b, 1986c, 1986d; Walker, Jones \& Savage 1986; Walker, Jones \& Savage 1989; Lane \& Heatwole 1991; O’Neill \& Heatwole 1996).

Seabirds play an important role in the ecology of coral cays. Deposition of guano and carrion drives nutrient cycling and has a major influence on soils and vegetation (Heatwole 1981). Seabirds also are important agents of plant dispersal (Heatwole \& Walker 1989; Heatwole 1991) and significant consumers of marine resources such as fish and squid (Harrison 1990).

The islands of the Swain Reefs constitute one of six core seabird breeding areas identified in Queensland (King 1993). Human visitation to islands of the Great Barrier Reef, such as the 
Swain Reefs cays, is a major concern for park managers (Stokes et al 1996). To devise an effective management plan for this valuable resource it is important to develop an understanding of the baseline fluctuations of the undisturbed populations. The present study was designed to fill that need. It was initiated when the Swain Reefs were rarely visited, and has continued up to the present as human influence is increasingly felt.

\section{STUDY AREA}

The Swain Reefs is a complex labyrinth of approximately 370 patch reefs lying between 120 kilometres and 250 kilometres off the coast of Queensland, Australia, near the southern end of the Great Barrier Reef (latitude $20^{\circ} 50^{\prime} \mathrm{S}-22^{\circ} 25^{\prime} \mathrm{S}$, longitude $151^{\circ} 15^{\prime} \mathrm{E}-153^{\circ} 00^{\prime} \mathrm{E}$ ) (Fig. 1). Covering an area of approximately 16,900 square kilometres, the Swain Reefs are wholly within the Great Barrier Reef Marine Park (GBRMP).

There are nine cays that regularly have breeding populations of seabirds and a number of smaller ones, including some incipient islands currently only emergent at low tides. These sand cays consist of coarse carbonate sand and bioclastic sediment of variable size. The major components are derived from coral, coralline algae, foraminiferans and molluscs, and display varying degrees of rounding and sorting (Flood \& Heatwole 1986).

The vegetation of the Swain Reefs cays can be transient. Since it was described in detail by Flood and Heatwole (1986), all vegetation has disappeared from Bacchi, Bylund, and Gannet Cays, leaving only Bell, Price and Frigate Cays with vegetative cover. On these three islands the vegetation is similar in both composition and structure. The most commonly occurring plants are Boerhavia diffusa and two grass species, Lepturus repens and Thuarea involuta. Coronopus integrifolius is common on Frigate and Price Cays, and Ipomea pescapre and Tribulus cistoides are well established on Bell Cay.

Impact from recreational use has been low in the Swain Reefs compared to most areas within the GBRMP, because of their geographical isolation. The area is currently beyond the range of one-day tourist vessels (Stokes et al. 1996), but human visitation increased between 1980 and 1990 as a result of long-range, tourist charter vessels operating out of the ports of Gladstone and Yeppoon. Tourists from these charter vessels, and crews from fishing vessels, are known to visit the cays from time to time. 
Figure 1 Location map of the Swain Reefs. 


\section{METHODS}

\subsection{Survey methods}

Numbers of seabirds and nests were recorded on seven Swain Reefs cays from 1982 to 1994. Cay names were taken from the Great Barrier Reef Marine Park Authority zoning maps. Factors that may influence population size; cay morphology, vegetation, climate, oceanographic conditions and levels of human visitation, were investigated.

For comparison of breeding phenology, counts were carried out twice yearly, once in summer and once in winter. Summer censuses spanned the months of November to February, but all winter censuses were carried out in July. All counts were done in the morning, with start times before 0900 hours. A number of different observers assisted in the counts. However, observer variability was minimised because at least one of the authors was present on all counts included in the analyses. Appendix 1 is a list of all ornithological trips to the area.

Upon arrival, and before other people went ashore, two observers circled the island in a dinghy and made independent counts of the birds on sections of the beaches. The observers then landed and completed the census on foot, by walking slowly from one end of the island to the other and counting all birds present above the high water mark. Section totals were compared between the two observers. If differences were less than five percent of the total, the counts were averaged. Larger discrepancies (rare) resulted in a recount.

Birds were recorded by age: adult, immature (fledged but before attaining adult plumage), juvenile (first pennaceous feathers up to fledging), downy chick, naked chick or egg. Numbers of nests and their contents were recorded for each breeding species. Disturbance of sitting birds was minimised, with nest contents usually estimated from observations using binoculars. Nests were only recorded where pairs were observed displaying territorial behaviour or where a nest structure, eggs or chicks were visible.

\subsection{Environmental measures}

The total area of the cays and their vegetated zones were mapped and measured from aerial photographs taken in July 1964, November 1984, October 1992 (Bell and Gannet Cays only) 
and October-November 1993 (except Gannet Cay). Measurements were made using a Keiffel and Esser compensating polar planimeter (Model 620,000). Coral patterns on the reef flats were used for control of relative map scale and orientation. Additional data were available from ground measurements made in July 1983, January 1984, July 1984, and January 1985 (from Heatwole 1987). Changes in location, size and shape through time of the seven most visited cays were examined.

Daily values of the Southern Oscillation Index (SOI), which are used as a measure of the strength of the El Niño Southern Oscillation (ENSO), and daily sea surface temperatures (SST's), were obtained for the area covering the Great Barrier Reef between latitudes $20^{\circ} \mathrm{S}$ and $22^{\circ} \mathrm{S}$ (Lough, 1994). Meteorological records (air temperature, wind speed and wind direction taken daily at 1300 hours from 1985 to 1995) from the automatic recording station at Gannet Cay, and maps of all cyclone tracks in Queensland from 1971 to 1995, were acquired from the Australian Bureau of Meteorology (ABM).

Information was sought that might indicate trends in levels of human visitation to the Swain Reefs area. Records of levels of use from permits issued by the Great Barrier Reef Marine Park Authority for activities in the area were examined, but found to be unsuitable because return of data by operators was sporadic prior to it becoming mandatory in 1992.

As part of the Great Barrier Reef Park surveillance program, aerial surveys were carried out monthly by the Queensland Department of Environment in the Swain Reefs from 1988 to 1995. The area was divided into a series of irregularly shaped plots for flight pogramming purposes, and a number of these plots were selected randomly for investigation before each flight. Two plots ( $F$ and $G$ ) cover the area of the cays, and the numbers of vessels from these two plots were extracted for analysis.

No data on fishing effort in the Swain Reefs during the period of the study could be located. However, some incidental observations are reported in the Results.

\subsection{Statistical analyses}

Temporal trends in the numbers of adult birds and nests of masked and brown boobies and adult silver gulls were investigated. Uncertainties associated with counting (see Discussion) 
precluded other species from these trend analyses, and they are reported in general terms only.

Census results were graphed for each of the three species and seven islands, and separately for summer and winter. Regional population trends were also investigated, by combining census results across cays and plotting onto "TOTALS" graphs. Data from Bell Cay were not included in these totals, because it was visited less regularly.

Counts were log-normal transformed, before fitting least-squares regression lines. Regression slopes were tested for significant differences from zero, using two-tailed $t$-tests. Changes in the combined ("TOTALS") counts were tested for significance by averaging the regression slopes calculated separately for each cay. This utilised all data collected, and avoided only testing the reduced data points available in the "TOTALS" graphs. Regression lines were fitted to the "TOTALS" graphs to aid visual interpretation. The means of the summer and winter counts (nests and adult birds) on each cay, were compared using Wilcoxon tests.

Smoothers (Hastie and Tibshirani 1990) were fitted to both the climate and vessel count data, as an aid to identifying trends through time. The smoothers, calculated using the cubic spline technique, consist of a series of third-degree polynomials spliced together to produce a continuous curve, which is smooth at the splices (knot points). The resultant line varies in smoothness according to the chosen value of lambda (l), a tuning parameter in the spline formula. Smoothers were fitted to illustrate either: a) within year trends; b) gradual, long-term trends; or c) trends over periods of several years (e.g. El Niño events). Lambda values were chosen visually after a series of trials to produce a reasonable compromise between flexibility and smoothness of fit, and to best illustrate the trends of interest. Any conclusions based on these trends are therefore not inferentially based, and must be viewed with caution. 


\section{RESULTS}

\subsection{Breeding Records}

Thirty-seven bird species were recorded in the Swain Reefs during the study and twelve of these were observed breeding (Table 1). The largest number of species (26) was recorded on Bell Cay, with 22 on Frigate, Gannet and Price Cays. Frigate Cay had the most breeding species (10), while nine were recorded on Bell Cay, and seven each on Price and Bacchi, although only three species have bred on Bacchi Cay since devegetation in the late 1980's. The only known loss of a breeding species in the Swain Reefs is the reef egret (Egretta sacra), which bred on the wreck of the "Swalong" on Horseshoe Reef before it disintegrated during a cyclone in 1993. Two species, sooty tern (Sterna fuscata) and bridled tern (Sterna anaethetus), have not been recorded breeding on Gannet Cay since the vegetation disappeared in 1983. 
Table 1 Status and location of bird species recorded in the Swain Reefs.

\begin{tabular}{|c|c|c|c|c|c|c|c|c|c|c|c|c|c|}
\hline \multirow[t]{2}{*}{ Species } & \multicolumn{13}{|c|}{ Cay } \\
\hline & $\mathrm{Bac}$ & Bel & Byl & Dis & Fri & Gan & Hix & Hor & Lav & Pri & Rip & Tho & Zod \\
\hline Giant-Petrel* ${ }^{*}$ & & & & & & & & & & + & & & \\
\hline Wedge-tailed Shearwater & + & + & & & + & + & & & & & & + & \\
\hline Fluttering Shearwater & & & & & & & & & & & & + & \\
\hline Australian Pelican & & + & & & & & & & & & & & \\
\hline Red-footed Booby & & & & & & + & & & & & & & \\
\hline Masked Booby & B & B & B & + & B & B & + & & & B & B & B & \\
\hline Brown Booby & B & B & B & B & $\mathrm{B}$ & B & + & B & + & B & B & B & + \\
\hline Australian Darter & & & & & & & & & & & & + & \\
\hline Great Frigatebird & + & + & & & + & & & + & & + & & + & \\
\hline Least Frigatebird & & B & & & B & + & & & + & + & + & + & + \\
\hline Red-tailed Tropicbird & & + & & & & & & & & & & & \\
\hline White-faced Heron & & + & & & + & & & & & + & & & \\
\hline Cattle Egret & & & & & & + & & & & + & & & \\
\hline Eastern Reef Egret & + & + & & & + & & & B & & + & & + & \\
\hline White-bellied Sea-Eagle & & + & & & & & & & & & & & \\
\hline Buff-banded Rail & & + & & & & & & & & & & & \\
\hline Lesser Golden Plover & + & + & + & & + & + & & & & + & + & & \\
\hline Ruddy Turnstone & + & + & + & + & + & + & + & + & & + & + & + & \\
\hline Whimbrel & & + & & & + & & & & & & & & \\
\hline Tattler spp.* & & + & & + & & + & & + & & & + & & \\
\hline Bar-tailed Godwit & + & & & & + & + & & & & + & & & \\
\hline Red-necked Stint & & & & & & + & & & & + & & & \\
\hline Sanderling** & & & & & + & & & & & & & & \\
\hline Skua* & & + & & & & & & & & & & & \\
\hline Silver Gull & B & B & B & + & B & B & + & B & + & B & + & B & \\
\hline Common Tern & & & + & & + & + & + & & & & & & \\
\hline Roseate Tern & + & + & B & + & B & + & + & + & & + & + & + & \\
\hline Black-naped Tern & B & + & B & B & B & B & + & B & + & + & + & B & + \\
\hline Sooty Tern & + & B & & & & + & + & & & & + & & \\
\hline Bridled Tern & + & B & + & & $\mathrm{B}$ & B & + & B & & B & & + & \\
\hline Little Tern & + & & + & + & + & + & + & & & + & + & + & \\
\hline Crested Tern & B & B & B & + & B & + & + & + & + & B & $\mathrm{B}$ & B & \\
\hline Lesser Crested Tern & B & B & + & & B & + & + & & & B & & B & \\
\hline Common Noddy & B & B & + & B & B & B & + & + & + & B & + & + & + \\
\hline Black Noddy & + & + & + & & + & + & + & + & & + & & + & \\
\hline Sacred Kingfisher & & + & & & & & & & & & & & \\
\hline Welcome Swallow & & & & & & & & & & + & & & \\
\hline Number of species & 18 & 26 & 14 & 10 & 22 & 22 & 14 & 12 & 6 & 22 & 13 & 18 & 4 \\
\hline $\begin{array}{l}\text { Number of breeding } \\
\text { species }^{\mathrm{a}}\end{array}$ & $7(3)$ & 9 & $6(4)$ & 3 & 10 & $6(3)$ & 0 & $5(0)$ & 0 & 7 & 3 & 6 & 0 \\
\hline
\end{tabular}

$+=$ recorded present on or near island, but not breeding; $\mathrm{B}=$ recorded breeding.

*Species unknown. **Sighting unconfirmed.

a The number of breeding species currently found on cays that have lost previous vegetative cover is reduced, and shown in brackets.

Bac=Bacchi Cay, Bel=Bell Cay, Byl=Bylund Cay, Dis=Distant Cay, Fri=Frigate Cay, Gan=Gannet Cay, Hix=Hixson Cay, 
Hor=Horsehoe Reef (wreck), Lav=Lavers Cay, Pri=Price Cay, Rip=Riptide Cay, Tho=Thomas Cay, Zod=Zodiac Cay. 
Approximate numbers of breeding pairs of each species recorded in the Swain Reefs during the period of the study are shown in Table 2 . These values have been divided into three time periods (pre-1985, 1985-1989, 1990-1994). Ranges within each time period indicate variation within each period.

Table 2 Estimated numbers of breeding pairs of seabirds present during their respective principal breeding seasons on Swain Reef cays, in the periods Pre 1985, 1985-1989 \& 1990-1994.

(Pre 1985)

\begin{tabular}{|c|c|c|c|c|c|c|c|c|c|c|}
\hline & Bacchi & Bell & Bylund & Distant & Frigate & Gannet & Price & Riptide & Thomas & Totals \\
\hline Masked booby & 2 & 2 & 8 & nd & 34 & 164 & 13 & 20 & 15 & 258 \\
\hline Brown booby & 0 & 5 & 0 & nd & 14 & 360 & 37 & 80 & 28 & 524 \\
\hline Lesser frigatebird & 0 & 44 & 0 & nd & + & 0 & 0 & 0 & 0 & 44 \\
\hline Silver gull & + & 1 & 1 & nd & $1-12$ & $3-7$ & 3 & 0 & 1 & $10-25$ \\
\hline Black-naped tern & 0 & 0 & 200 & nd & $<150$ & 0 & 0 & 0 & 0 & $<350$ \\
\hline Sooty tern & 0 & 0 & 0 & nd & 0 & 0 & 0 & 0 & 0 & $\mathbf{0}$ \\
\hline Bridled tern & $>200$ & 0 & 0 & nd & $\begin{array}{l}100- \\
200\end{array}$ & $\begin{array}{l}150- \\
164\end{array}$ & $12-50$ & 0 & 0 & 462-614+ \\
\hline Crested tern & 0 & $82-102$ & $20+$ & nd & $\begin{array}{l}100- \\
500\end{array}$ & 0 & 500 & 400 & 13 & $\begin{array}{l}1115- \\
1535\end{array}$ \\
\hline $\begin{array}{l}\text { Lesser crested } \\
\text { tern }\end{array}$ & 0 & 0 & 0 & nd & 0 & 0 & 9 & 0 & 0 & 9 \\
\hline Common noddy & $0-4$ & $46-400$ & 0 & nd & $\begin{array}{l}200- \\
800\end{array}$ & $27-324$ & 20 & 0 & 0 & 293-1548 \\
\hline
\end{tabular}

$(1985-1989)$

\begin{tabular}{lllllllllll}
\hline & Bacchi & Bell & Bylund & Distant & Frigate & Gannet & Price & Riptide & Thomas & Totals \\
\hline Masked booby & $3-7$ & $1-5$ & $5-29$ & 0 & $11-57$ & $40-183$ & $22-51$ & $30-40$ & $1-26$ & $\mathbf{1 1 3 - 3 9 8}$ \\
Brown booby & $51-82$ & $30-40$ & $7-39$ & $20+$ & $39-344$ & $30-288$ & $36-316$ & $100-$ & $23-95$ & $\mathbf{3 3 6 - 1 4 2 4}$ \\
& & & & & & & & 200 & & \\
Lesser frigatebird & 0 & $22-155$ & 0 & 0 & 0 & 0 & 0 & 0 & 0 & $\mathbf{2 2 - 5 5}$ \\
Silver gull & 1 & $3-9$ & $2-4$ & $2-4$ & $1-4$ & $2-10$ & $1-6$ & 0 & $1-7$ & $\mathbf{1 3 - 4 5}$ \\
Black-naped tern & $0-24$ & 0 & + & $0-5$ & 0 & $0-10$ & 0 & 0 & $0-24$ & $\mathbf{0 - 6 3}$ \\
Sooty tern & 0 & $10-50$ & 0 & 0 & 0 & 0 & 0 & 0 & 0 & $\mathbf{1 0 - 5 0}$ \\
Bridled tern & 0 & $0-2$ & $1-70$ & 0 & 0 & 0 & $2-45$ & 0 & 0 & $\mathbf{3 - 1 1 7}$ \\
Crested tern & $9-200$ & $18-190$ & 0 & 0 & $0-1200$ & 0 & $30-500$ & 0 & $100-$ & $\mathbf{1 5 7 - 2 4 0 2}$ \\
Lesser crested & $0-4$ & $0-1$ & $0-100$ & 0 & 0 & 0 & $0-230$ & 0 & $0-163$ & $\mathbf{0 - 4 9 8}$ \\
tern & & & & & & & & & & $\mathbf{1 0 6 2 -}$ \\
Common noddy & $1-5$ & $100-$ & 0 & 15 & $3-600$ & 0 & $43-616$ & 0 & 0 & $\mathbf{2 4 3 6}$ \\
\hline
\end{tabular}

(1990-1994)

\begin{tabular}{lllllllllll}
\hline & Bacchi & Bell & Bylund & Distant & Frigate & Gannet & Price & Riptide & Thomas & Totals \\
\hline Masked booby & $<5$ & $2-10$ & $2-17$ & 0 & $10-43$ & $34-95$ & $8-50$ & $30-40$ & $4-14$ & $\mathbf{9 5 - 2 7 4}$ \\
Brown booby & $2-17$ & $7-24$ & $1-3$ & nd & $16-129$ & $11-118$ & $5-101$ & $100-200$ & $2-18$ & $\mathbf{1 4 4 - 6 1 0}$ \\
Lesser frigatebird & 0 & $22-$ & 0 & 0 & 0 & 0 & 0 & 0 & 0 & $\mathbf{2 2 - 1 0 0}$ \\
& & $100+$ & & & & & & & & \\
Silver gull & $1-2$ & $1-8$ & $0-1$ & $0-1$ & $0-1$ & $2-3$ & $0-3$ & 0 & 0 & $\mathbf{4 - 1 9}$ \\
Black-naped tern & $0-1$ & 0 & $5-50$ & 0 & 0 & 0 & 0 & 0 & 0 & $\mathbf{5 - 5 1}$
\end{tabular}




\begin{tabular}{lllllllllll} 
Sooty tern & 0 & $10-50$ & 0 & 0 & 0 & 0 & 0 & 0 & 0 & $\mathbf{1 0 - 5 0}$ \\
Bridled tern & 0 & $0-100+$ & $0-150+$ & 0 & 0 & 0 & $0-100+$ & 0 & 0 & $\mathbf{0 - 3 5 0 +}$ \\
Crested tern & 0 & $1-410$ & $<20$ & 0 & $0-200$ & 0 & $0-200$ & $0-1200$ & $0-1$ & $\mathbf{2 0 - 2 0 3 0}$ \\
Lesser crested & 0 & 0 & $0-80$ & 0 & 0 & 0 & $0-77$ & 0 & 0 & $\mathbf{0 - 1 5 7}$ \\
tern & & & & & & & & & & \\
Common noddy & 0 & $100-$ & 0 & 0 & $200-$ & 0 & $100-$ & 0 & 0 & $\mathbf{4 0 0 -}$ \\
& & 200 & & & 1100 & & 400 & & & $\mathbf{1 4 0 0}$ \\
\hline
\end{tabular}

nd $=$ no data available, $+=$ present, but no numbers recorded . 


\subsubsection{Brown booby (Sula leucogaster)}

Significant declines $(\mathrm{p}<0.05)$ in the numbers of brown booby nests occurred on four of the seven cays between 1983 and 1993 (Table 3). There is strong evidence that a major decline $(p=0.002$, Table 4$)$ in the combined numbers of nests counted in summer also occurred (“TOTALS" graph in Fig. 2).

Table 3 Statistical difference from zero for slopes of regression lines fitted to summer and winter counts of adults and nests of brown and masked boobies, and adults of silver gulls. All data are log-normal transformed. ns = not significant.

\begin{tabular}{|c|c|c|c|c|c|c|c|c|c|c|c|}
\hline \multirow{3}{*}{ Cay } & & \multicolumn{4}{|c|}{ Brown booby } & \multicolumn{4}{|c|}{ Masked booby } & \multicolumn{2}{|c|}{ Silver gull } \\
\hline & & \multicolumn{2}{|c|}{ summer } & \multicolumn{2}{|c|}{ winter } & \multicolumn{2}{|c|}{ summer } & \multicolumn{2}{|c|}{ winter } & \multirow{2}{*}{\begin{tabular}{|c} 
summer \\
adults
\end{tabular}} & \multirow{2}{*}{$\frac{\text { winter }}{\text { adults }}$} \\
\hline & & adults & nests & adults & nests & adults & nests & adults & nests & & \\
\hline \multirow[t]{3}{*}{ Bacchi } & $t$ & -2.48 & -2.66 & -1.79 & 1.62 & 1.43 & 0.87 & 0.11 & 0.79 & -2.95 & -3.51 \\
\hline & $d f$ & 10 & 4 & 9 & 4 & 4 & 3 & 5 & 4 & 10 & 9 \\
\hline & $P$ & 0.033 & $\mathrm{~ns}$ & $\mathrm{~ns}$ & $\mathrm{~ns}$ & $\mathrm{~ns}$ & $\mathrm{~ns}$ & $\mathrm{~ns}$ & ns & 0.015 & 0.007 \\
\hline \multirow[t]{3}{*}{ Bell } & $t$ & 0.10 & -6.35 & 0.73 & -0.66 & 1.81 & 4.14 & 3.36 & 1.91 & -2.97 & -0.09 \\
\hline & $d f$ & 8 & 1 & 1 & 4 & 1 & 1 & 7 & 6 & 1 & 8 \\
\hline & $P$ & $\mathrm{~ns}$ & $\mathrm{~ns}$ & $\mathrm{~ns}$ & $\mathrm{~ns}$ & $\mathrm{~ns}$ & $\mathrm{~ns}$ & 0.012 & $\mathrm{~ns}$ & $\mathrm{~ns}$ & $\mathrm{~ns}$ \\
\hline \multirow[t]{3}{*}{ Bylund } & $t$ & -4.24 & -1.49 & -1.30 & -1.53 & -0.33 & 1.60 & -0.44 & -1.91 & -10.90 & -2.95 \\
\hline & $d f$ & 9 & 6 & 9 & 6 & 9 & 4 & 9 & 8 & 7 & 9 \\
\hline & $P$ & 0.002 & $\mathrm{~ns}$ & $\mathrm{~ns}$ & $\mathrm{~ns}$ & $\mathrm{~ns}$ & $\mathrm{~ns}$ & $\mathrm{~ns}$ & $\mathrm{~ns}$ & 0.000 & 0.016 \\
\hline \multirow[t]{3}{*}{ Frigate } & $t$ & -4.52 & -3.88 & 0.21 & 1.90 & 0.90 & 1.51 & -0.04 & -0.44 & -0.80 & -0.96 \\
\hline & $d f$ & 9 & 5 & 8 & 5 & 9 & 5 & 9 & 6 & 9 & 9 \\
\hline & $P$ & 0.001 & 0.012 & $\mathrm{~ns}$ & $\mathrm{~ns}$ & ns & $\mathrm{ns}$ & $\mathrm{ns}$ & ns & $\mathrm{ns}$ & ns \\
\hline \multirow[t]{3}{*}{ Gannet } & $t$ & -4.66 & -4.84 & -1.51 & 0.78 & -1.08 & -0.64 & -1.08 & -0.21 & -0.11 & -4.90 \\
\hline & $d f$ & 10 & 8 & 9 & 8 & 11 & 5 & 10 & 8 & 11 & 10 \\
\hline & $P$ & 0.001 & 0.001 & $\mathrm{~ns}$ & $\mathrm{~ns}$ & $\mathrm{~ns}$ & $\mathrm{~ns}$ & $\mathrm{~ns}$ & $\mathrm{~ns}$ & $\mathrm{~ns}$ & 0.001 \\
\hline \multirow[t]{3}{*}{ Price } & $t$ & -3.65 & -3.01 & 0.53 & 1.23 & 2.46 & 5.07 & 0.21 & -0.59 & -0.25 & 0.69 \\
\hline & $d f$ & 9 & 7 & 8 & 5 & 9 & 5 & 8 & 6 & 10 & 7 \\
\hline & $P$ & 0.005 & 0.019 & $\mathrm{~ns}$ & $\mathrm{~ns}$ & 0.036 & 0.004 & $\mathrm{~ns}$ & $\mathrm{~ns}$ & $\mathrm{~ns}$ & ns \\
\hline \multirow[t]{3}{*}{ Thomas } & $t$ & -2.09 & -4.67 & -0.17 & 0.26 & -0.30 & -0.92 & 1.11 & -2.17 & -0.49 & -1.28 \\
\hline & $d f$ & 10 & 6 & 9 & 5 & 10 & 4 & 9 & 7 & 9 & 9 \\
\hline & $P$ & $\mathrm{~ns}$ & 0.003 & $\mathrm{~ns}$ & $\mathrm{~ns}$ & $\mathrm{~ns}$ & $\mathrm{~ns}$ & $\mathrm{~ns}$ & $\mathrm{~ns}$ & $\mathrm{~ns}$ & $\mathrm{~ns}$ \\
\hline
\end{tabular}


Table 4 Slopes of regression lines fitted to counts of adults and nests of brown and masked boobies and adults of silver gulls for each cay. Statistical difference from zero is provided for the mean slopes of regression lines for all cays. All data are log-normal transformed. $\mathrm{ns}=$ not significant .

\begin{tabular}{|c|c|c|c|c|c|c|c|c|c|c|}
\hline \multirow{3}{*}{ Cay } & \multicolumn{4}{|c|}{ Brown booby } & \multicolumn{4}{|c|}{ Masked booby } & \multicolumn{2}{|c|}{ Silver gull } \\
\hline & \multicolumn{2}{|c|}{ summer } & \multicolumn{2}{|c|}{ winter } & \multicolumn{2}{|c|}{ summer } & \multicolumn{2}{|c|}{ winter } & \multirow{2}{*}{$\begin{array}{c}\text { summer } \\
\text { adults } \\
\end{array}$} & \multirow{2}{*}{$\begin{array}{l}\text { winter } \\
\text { adults } \\
\end{array}$} \\
\hline & adults & nests & adults & nests & adults & nests & adults & nests & & \\
\hline Bacchi & -0.0075 & -0.0115 & -0.0031 & -0.011 & 0.0112 & 0.006 & 0.0004 & 0.0055 & -0.0065 & -0.0043 \\
\hline Bell & 0.0002 & -0.0033 & 0.0012 & -0.0034 & 0.001 & 0.0034 & 0.0057 & 0.0045 & -0.008 & -0.0001 \\
\hline Bylund & -0.0114 & -0.0087 & -0.0044 & -0.0042 & -0.0007 & 0.0046 & -0.0006 & -0.0045 & -0.0103 & -0.0051 \\
\hline Frigate & -0.0049 & -0.0111 & 0.0004 & 0.0117 & 0.0029 & 0.0057 & -0.0001 & -0.0013 & -0.0021 & -0.001 \\
\hline Gannet & -0.008 & -0.0231 & -0.0019 & 0.0036 & -0.0037 & -0.0092 & -0.0014 & -0.0007 & -0.0002 & -0.005 \\
\hline Price & -0.0058 & -0.0192 & 0.0012 & 0.0063 & 0.0057 & 0.0138 & 0.0003 & -0.0019 & -0.0005 & -0.0013 \\
\hline Thomas & -0.0055 & -0.0135 & -0.0004 & 0.0013 & -0.0017 & -0.0031 & 0.002 & -0.0043 & -0.0013 & -0.0019 \\
\hline mean & -0.006 & -0.013 & -0.001 & 0.001 & 0.002 & 0.003 & 0.001 & -0.000 & -0.004 & -0.003 \\
\hline$t$ & -4.579 & -5.200 & -1.208 & 0.216 & 1.098 & 1.094 & 1.009 & -0.258 & -2.683 & -3.405 \\
\hline$d f$ & 5 & 5 & 5 & 5 & 5 & 5 & 5 & 5 & 5 & 5 \\
\hline$P$ & 0.004 & 0.002 & ns & $\mathrm{ns}$ & $\mathrm{ns}$ & $\mathrm{ns}$ & ns & $\mathrm{ns}$ & 0.036 & 0.014 \\
\hline
\end{tabular}


Brown boobies were present on the cays during all months visited. Numbers nesting were usually lower in winter (July) than in the summer months (Fig. 2). However, because numbers of summer nesters declined below numbers of winter nesters in later years, the means of the winter and summer counts on each cay were mostly not significantly different. The exceptions were Bacchi and Thomas Cays, where the means of the winter counts remained significantly lower than the means of the summer counts ( $p<0.05$, Table 5). Means of the combined counts were not significantly different between winter and summer (Table 5). The brown booby did, however, have a clear summer nesting preference on all cays during the earlier part of the project (Fig. 2).

Table 5 Comparison (chi-squared tests) of means of summer and winter counts of adults and nests of brown and masked boobies, and adults of silver gulls. The "TOTALS" row refers to the combined data in the "TOTALS" graphs (Figs. 2 to 6), and excludes Bell Cay and counts not completed for all of the other six cays. ns $=$ not significant.

\begin{tabular}{|c|c|c|c|c|c|c|}
\hline \multirow[t]{2}{*}{ Cay } & & \multicolumn{2}{|c|}{ Brown booby } & \multicolumn{2}{|c|}{ Masked booby } & \multirow{2}{*}{$\begin{array}{c}\text { Silver gull } \\
\text { adults } \\
\end{array}$} \\
\hline & & adults & nests & adults & nests & \\
\hline \multirow[t]{3}{*}{ Bacchi } & $X^{2}$ & 3.187 & 4.021 & 0.084 & 0.009 & 1.107 \\
\hline & $d f$ & 1 & 1 & 1 & 1 & 1 \\
\hline & $P$ & ns & 0.045 & ns & ns & ns \\
\hline \multirow[t]{3}{*}{ Bell } & $X^{2}$ & 1.029 & 1.361 & 1.455 & 0.044 & 0.029 \\
\hline & $d f$ & 1 & 1 & 1 & 1 & 1 \\
\hline & $P$ & $\mathrm{~ns}$ & $\mathrm{~ns}$ & $\mathrm{~ns}$ & $\mathrm{~ns}$ & ns \\
\hline \multirow[t]{3}{*}{ Bylund } & $X^{2}$ & 2.701 & 0.732 & 0.312 & 5.479 & 0.072 \\
\hline & $d f$ & 1 & 1 & 1 & 1 & 1 \\
\hline & $P$ & ns & ns & ns & 0.019 & ns \\
\hline \multirow[t]{3}{*}{ Frigate } & $X^{2}$ & 2.085 & 3.433 & 4.148 & 8.070 & 0.853 \\
\hline & $d f$ & 1 & 1 & 1 & 1 & 1 \\
\hline & $P$ & $\mathrm{~ns}$ & ns & 0.042 & 0.005 & $\mathrm{~ns}$ \\
\hline \multirow[t]{3}{*}{ Gannet } & $X^{2}$ & 2.661 & 1.050 & 0.265 & 4.369 & 0.127 \\
\hline & $d f$ & 1 & 1 & 1 & 1 & 1 \\
\hline & $P$ & $\mathrm{~ns}$ & $\mathrm{~ns}$ & $\mathrm{~ns}$ & 0.037 & $\mathrm{~ns}$ \\
\hline \multirow[t]{3}{*}{ Price } & $X^{2}$ & 1.044 & 0.070 & 4.768 & 6.247 & 0.005 \\
\hline & $d f$ & 1 & 1 & 1 & 1 & 1 \\
\hline & $P$ & $\mathrm{~ns}$ & $\mathrm{~ns}$ & 0.029 & 0.012 & $\mathrm{~ns}$ \\
\hline \multirow[t]{3}{*}{ Thomas } & $X^{2}$ & 2.761 & 5.120 & 3.534 & 9.096 & 6.276 \\
\hline & $d f$ & 1 & 1 & 1 & 1 & 1 \\
\hline & $P$ & ns & 0.024 & ns & 0.003 & 0.012 \\
\hline \multirow[t]{3}{*}{ TOTALS } & $X^{2}$ & 5.078 & 3.429 & 3.617 & 5.346 & 0.031 \\
\hline & $d f$ & 1 & 1 & 1 & 1 & 1 \\
\hline & $P$ & 0.024 & ns & ns & 0.021 & $\mathrm{~ns}$ \\
\hline
\end{tabular}


All winter records were taken in July, and no particular temporal patterns emerged (Fig. 2). A slight increase evident in the combined winter numbers ("TOTALS" graph, Fig. 2) was not significant ( $>0.05$, Table 5). However, winter breeding activity increased to a level slightly above the much reduced summer breeding by the later part of the study. While this may indicate a slight shift in total breeding effort from summer toward the winter months, it was not enough to offset the summer losses.

Nesting densities for the brown booby were highly variable between cays (Table 6). Of the major breeding islands, the highest nest density recorded was a pair every 46 square metres on Gannet Cay, with the lowest density of one pair every 473 square metres recorded on Bell Cay.

Table 6 Minimum area $\left(\mathrm{m}^{2}\right)$ of cay above High Water mark recorded per nest site between 1985 and 1995, for the primary brown and masked booby breeding cays in the Swain Reefs.

\begin{tabular}{lcc}
\hline \multirow{2}{*}{ Cay } & \multicolumn{2}{c}{ Minimum area $\left(\mathrm{m}^{2}\right)$ per nest site recorded between 1985 and 1995} \\
\cline { 2 - 3 } & Brown booby & Masked booby \\
\hline Bell & 473 & 1420 \\
Frigate & 68 & 422 \\
Gannet & 46 & 137 \\
Price & 54 & 216 \\
\hline
\end{tabular}

\subsubsection{Masked booby (Sula dactylatra)}

No significant trends in masked booby nest numbers were recorded, except on Price Cay where a summer increase occurred (Fig. 3, Table 3). On most cays, winter nest numbers remained relatively stable; the slight winter decline ("TOTALS" graph Fig. 3), was not significant (Table 4).

On most islands, the mean number of nests counted in winter was significantly higher than the mean number counted in summer. Exceptions were Bacchi and Bell Cays, where no difference was detected (Table 5). The mean of the combined winter nest counts was significantly higher than the summer mean (Table 5), indicating a clear winter nesting preference for the masked booby in the Swain Reefs (“TOTALS" graph in Fig. 3).

The pattern of nesting densities for the masked booby was similar to that of the brown booby, although the brown booby nest densities were much higher in absolute terms (Table 6). The 
highest density, of one pair every 137 square metres, was recorded on Gannet Cay. The lowest density was on Bell Cay, with one pair every 1420 square metres.

\subsubsection{Silver gull (Larus novaehollandiae)}

Silver gulls were recorded breeding on 47 occasions (140 nests) in the Swain Reefs' cays. Of these, 32 (99 nests) were recorded in July, six (13 nests) in April, and only nine (28 nests) in summer months. As these records are from 13 winter trips, two April trips, and 15 summer trips, counting effort was similar between summer and winter. The nest records, therefore, indicate a clear preference for winter nesting by the silver gull in the Swain Reefs. 
The silver gull was recorded nesting on most islands, but only in scattered pairs. For this reason nests were sometimes difficult to locate and it is possible that some may have been missed. These data were not, therefore, analysed for trends. Only small numbers were ever counted and no trends were observed.

\subsubsection{Other species}

Lesser frigatebirds (Fregata ariel) historically nested on Frigate Cay, but the last nesting record from there was in 1962 (McMichael 1963). In November 1976, Limpus and Lyon (1981) recorded 44 lesser frigatebird nests on Bell Cay (Table 2). Egg laying on Bell Cay occurs mainly from January to April, with most young either still downy or partly feathered by July. The numbers of nests recorded fluctuated widely between years, supporting the hypothesis that lesser frigatebirds do not breed every year because of the protracted nature of their chick dependence and their habit of delaying moult until after breeding (Lindsey 1986). The highest number of nests recorded at Bell Cay was 155 in July 1986, and in March 1994 the colony showed relative stability with more than 100 nests recorded. The colony was extremely vulnerable to egg predation by silver gulls when adult frigatebirds arose from the nest if people approached closely or carelessly.

There are only three records of roseate terns (Sterna dougallii) nesting in the Swain Reefs, twice on Bylund Cay (November 1976 and July 1989) and once on Frigate Cay (April 1986). The 1989 record was based on the mobbing behaviour of a single pair. The 1976 record from Bylund Cay (Limpus and Lyon 1981) documents only two nests with eggs, and the 1986 Frigate Cay record was of a single nest.

The black-naped tern (Sterna sumatrana) was recorded nesting on most of the major seabird breeding cays in the Swain Reefs, exceptions being Bell and Price (Table 1), although the one breeding record from Frigate Cay is unconfirmed. Breeding black-naped terns were most frequently recorded at Bylund Cay, where the size of the nesting colony was highly variable. Approximately 200 nests were present in November 1976, and more recently about 50 in November 1991. Breeding records from other locations (7) were all fewer than 25 nests, and most came from islands with little or no vegetative cover. There were a number of records of up to 17 nests on the wreck of the Taiwanese fishing vessel 'Swalong' on Horseshoe Reef, but that wreck has since disintegrated. 
The sooty tern (Sterna fuscata) was recorded nesting only twice. As these two records (Bell Cay, February 1986 and July 1993), were of small numbers nesting amongst bridled terns (Sterna anaethetus), it is possible this species was sometimes overlooked.

Bridled terns (Sterna anaethetus) were recorded breeding on all densely vegetated cays and on the wreck of the "Swalong" on Horseshoe Reef. They ceased to breed on Gannet Cay after the disappearance of the vegetation (last breeding record is 1979), but continue to nest on Bell, Frigate and Price Cays (Table 2). Recent records of more than 100 breeding pairs on each of these three islands are larger than any prior records, and there is no indication of the breeding numbers declining in the area. All 28 breeding records occurred in summer, between November and February.

Crested terns (Sterna bergii) were recorded breeding on most Swain Reefs cays; however, the species preferred some islands over others. Crested terns mostly formed dense breeding colonies in association with lesser crested terns (Sterna bengalensis). These mixed colonies were clearly segregated by species within their outer boundaries. The most favoured islands were Price, Frigate, Bell and Riptide Cays (Table 2). Single colonies as large as 1,200 breeding pairs were recorded (e.g. Frigate Cay, July 1989), but in some years a number of smaller colonies were located on different cays. The total breeding population was generally between 400 and 1200 pairs, with no indication of decline.

Smaller numbers of lesser crested terns than crested terns were observed breeding in the Swain Reefs (Table 2). Three colonies of over 100 nesting pairs were observed between 1985 and 1990, with one colony containing about 230 pairs. Since 1990 the largest nesting colony was about 80 pairs. As with the crested terns, lesser crested terns did not breed at the same time year after year, or on the same cay, and it was therefore difficult to ascertain if change occurred in the population size. Also, because of the irregular nature of breeding events, and the relatively short time required for breeding (about two months for incubation and fledging), it is probable that much breeding passed unobserved in the Swain Reefs. Both species tended to nest on the larger islands where greater numbers of silver gulls occur, and both were found to be susceptible to egg and chick predation by gulls if disturbed.

The breeding season of the common noddy (Anous stolidus) in the Swain Reefs peaked between October and March, although a small number of birds were usually sighted on nests 
during the winter months. Nests were built on or amongst the low vegetation and the species did not nest on the unvegetated cays. Up to about 1800 nesting pairs were recorded on Bell, Frigate and Price Cays at any one time, but the population fluctuated, with higher counts recorded between 1985 and 1990 than during the periods immediately before and after (Table 2). The size of the breeding population in 1994 was similar to that recorded prior to 1985 . Common noddies did not nest in any numbers on Bacchi Cay after 1988, or on Gannet Cay after 1984, which coincided with the loss of vegetative cover from those islands. 
The only other species recorded nesting in the Swain Reefs was the eastern reef egret (Egretta sacra). Two nests were recorded on the wreck of the "Swalong" on Horseshoe Reef in January of 1990.

\subsection{Numbers of birds}

The total numbers of adult birds of all species present on each island (breeding and nonbreeding) were recorded during the course of the study.

\subsubsection{Brown booby}

Temporal trends in the numbers of adult brown boobies counted generally followed that of the numbers of nests (compare Fig. 4 with Fig. 2). Significant declines in the numbers of adults counted in summer occurred on all cays except Bell and Thomas (Table 3). The largest declines, in real terms, occurred on Gannet, Frigate and Price cays. A highly significant decline in the total numbers of birds counted in summer was observed (Table 4). This decline lagged behind the similar decline in nest numbers, by between two and three years (compare “TOTALS” graphs in Figs. 2 and 4). No significant trends in the much smaller winter numbers were detected (Tables 3 and 4).

The numbers of adult brown boobies present on most cays were higher in summer than in winter prior to 1990 (Fig. 4), although the means of the summer counts were not significantly higher than the mean winter counts on any island. However, the mean of the combined summer counts was significantly higher than was the mean of the combined winter counts (Table 5). The combined summer mean was higher than the combined winter mean because the combined summer counts prior to 1987 were two to three times higher than the corresponding winter counts.

The total number of brown booby adults (breeding and non-breeding) in the Swain Reefs was estimated at approximately 1,300 birds. This estimate was based on the most complete recent count (July 1993) and made allowance for some birds at Riptide Cay (November 1992 count), and Distant Cay (January 1989 count). Approximately 800 of these birds were breeding and 600 were non-breeding adults. This can be compared to the February 1986 count of 3,200 adults, consisting of about 2,500 breeding and 700 non-breeding adults. 


\subsubsection{Masked booby}

No trends in numbers of masked boobies over the period of the study were evident on most islands. Significant increases were detected, however, in the numbers on Price Cay in summer, and Bell Cay in winter (Table 3). The slight declines in both the combined summer and combined winter numbers ("TOTALS" graph, Fig. 5), are not statistically significant (Table 4).

Mean numbers of masked boobies on each island were similar in summer and winter except on Price and Frigate Cays, where the mean number of adults present during winter was significantly higher than the mean number present during the summer months (Table 5). This contrasts with the consistent and significant overall differences in nest numbers counted between winter and summer reported above.

The July 1993 count indicated that there were approximately 600 masked boobies breeding in the Swain Reefs. An additional 400 non-breeding adults brings the total adult population to approximately 1100 birds. The data suggest that this total changed little during the period of the study (Table 2).

\subsubsection{Silver gull}

Silver gull numbers on the Swain Reefs showed a significant decline over the study period (Fig. 6, Table 4), similar to that of the brown booby (Fig. 2). This decline can be largely attributable to the significant reductions in numbers on the unvegetated cays. Bacchi, Bylund and Gannet Cays recorded significant winter declines (Table 3). Bacchi and Bylund Cays also recorded significant declines in the summer numbers (Table 3). No significant change was recorded in the numbers of silver gulls on the three cays that retained vegetative cover for the duration of the study (right hand graphs, Fig. 6), although numbers fluctuated from year to year.

Thomas was the only cay where a significant difference was recorded in the mean number of silver gulls between winter and summer, with a higher mean number recorded in winter. However, no significant difference was recorded between the mean of the combined summer counts and the mean of the combined winter counts (Table 5). 
In 1994, the population of silver gulls on all cays in the Swain Reefs was about 100 individuals. This number was a substantial reduction from the 150 birds counted in 1986.

\subsubsection{Other species}

The numbers of adult frigatebirds recorded in the Swain Reefs varied greatly between expeditions, perhaps indicating this species travels widely when not breeding. The highest numbers recorded were 200 in January 1988, and 161 in March 1994. The current population is probably about 200 adults, all situated on Bell Cay.

Large groups of non-breeding adult roseate terns are often found in the Swain Reefs. The largest numbers recorded were 15,500 in February 1985, 6,200 in January 1987 and 8,700 in January 1989. Since 1990, over 3,000 roseate terns were recorded on two occasions, but this was less than the very large numbers recorded prior to 1990. Although numbers of more than 1,000 were sighted in July, all of the largest counts occurred during the summer months.

The highest number of black-naped terns recorded in the Swain Reefs was 5,250 in July 1988. More than 1,500 birds were recorded three other times prior to 1990 (November 1976, July 1985 and January 1989). Only one recording of this magnitude has occurred in the years since, when 1,970 were recorded in November 1991. Large numbers are likely to be located in the area during any month, perhaps indicating that the population is largely resident, but sometimes out feeding when the counts were done. Alternatively, the population may be subject to irruptions from influxes of birds from other areas, or from a successful breeding/recruitment season.

All records of the bridled tern occurred in the summer months except one, a single bird observed on Bell Cay in July 1986. The largest number recorded was 1300 in November 1976, but since that time the totals were relatively stable between 300 and 500 birds.

In January 1989, a large total of 5,270 crested terns were counted in the Swain Reefs. In most other years the total number was between 1,000 and 2,000 birds. The population appears to be stable. Roughly equal numbers were likely to be encountered in any month, indicating that this species is probably also resident in the area. 
Lesser crested terns were also present all year in the Swain Reefs. Total numbers of adults were between 100 and 350, with no apparent trend over the study period. 
While common noddies also occurred in the area all year, much larger numbers were found in the summer months than in July. The largest number reported was 8,860 in April 1989, which did not include a count on Bell Cay. Most frequently, however, counts were between 2,000 and 4,000 birds. There was no evidence of declining numbers.

White-faced herons (Egretta novaehollandiae) were recorded consistently in the Swain Reefs, but only in July. The numbers increased slightly during the study period, and between 40 and 60 birds were recorded on most visits after 1992. All records of more than eight birds were from Bell Cay, where they were often observed roosting in a single flock on the beach. They were also observed on the reef flat, apparently feeding in the shallow pools.

Black noddies (Anous minutus) were observed in small numbers during most trips. Occasionally more than 1000 were seen, with much larger numbers roosting on the islands at night. Individuals were most often recorded on Gannet Cay, where they used the tower of the automatic weather station as a roosting site. A mass moulting by this species occurred on Thomas Cay in July 1995, with the island covered in black feathers from thousands of vigorously preening individuals.

Little terns (Sterna albifrons) were recorded sporadically during the course of the study. Numbers of between 100 and 200 were common in the area during summer, with only a single observation (45 birds) in July. All records of birds in breeding plumage occurred during March or April, but no breeding was observed. No little terns were sighted on Bell Cay, although they were regularly observed on all other major cays.

Ruddy turnstones (Arenaria interpres) were found in the area throughout the year, but larger numbers (120-160 birds) were recorded in the summer months.

The other bird species recorded in the Swain Reefs (Table 1) occurred either as vagrants or in very low numbers, but several of these were seen regularly. The eastern reef egret (Egretta sacra) was found in small numbers, mostly during the winter months. The pacific golden plover (Pluvialis fulva) was seen in all months, with larger numbers during summer. A total of 60 birds were recorded in February 1986. A lone buff-banded rail (Gallirallus philippensis), noted as absent from the Swain Reefs by Limpus and Lyon (1981), was observed a number of times on Bell Cay. Wedge-tailed shearwaters (Puffinus pacificus) 
were often noted in flocks on the surface of the sea or flying along wave crests, but none was recorded on the cays. Of the eleven sightings of tattlers in the area, only five were identified to species le vel. These were all recorded as grey-tailed tattlers (Heteroscelus brevipes) and a maximum number of 57 was recorded on Bell Cay in March 1994.

Table 7 Cyclones passing within 100 kilometres of the Swain Reefs in the period 1971 to 1995. Asterisks indicate those cyclones whose centre passed directly over the area.

\begin{tabular}{c|c|c|c}
\hline Cyclone & $\begin{array}{c}\text { Barometric pressure } \\
(\mathrm{mb})\end{array}$ & \multicolumn{2}{c}{$\begin{array}{c}\text { Date near Swain Reefs } \\
\begin{array}{c}\text { Wind speed recorded at 1 pm at } \\
\text { Gannet Cay (kms/hr) }\end{array}\end{array}$} \\
\hline Emily* & 853 & 1.4 .72 & $\mathrm{n} / \mathrm{a}$ \\
Alice & 1280 & 20.3 .74 & $\mathrm{n} / \mathrm{a}$ \\
David* & 813 & 18.1 .76 & 25.7 \\
Dawn & 1200 & 5.3 .76 & 10.3 \\
Watorea * & 1067 & 26.4 .76 & 15.4 \\
Kerry & 1253 & 2.3 .79 & 15.4 \\
Paul & 1267 & 6.1 .80 & 0 \\
Simon & 1000 & 5.3 .80 & 12.9 \\
Elinor & 1160 & 4.3 .83 & $\mathrm{n} / \mathrm{a}$ \\
Lance* & 1267 & 7.4 .84 & $\mathrm{n} / \mathrm{a}$ \\
Pierre & 1200 & 22.2 .85 & $\mathrm{n} / \mathrm{a}$ \\
Fran* & 1067 & 14.3 .92 & 12.4 \\
Rewa * & 1000 & 26.2 .94 & 7.2 \\
\hline
\end{tabular}

\subsection{Weather conditions and patterns of change on islands}

Thirteen cyclones passed within 100 kilometres of the Swain Reefs in the period 1971 to 1995 , and on six of these occasions the centre passed directly over the area. Table 7 lists these cyclones, their barometric pressure, the dates they passed closest to the Swain Reefs and the highest wind speed recorded at Gannet Cay at $1 \mathrm{pm}$ on the day each was closest to the area.

Only two cyclones, Emily (1972) and David (1976), had barometric pressures below 1000 millibars when close to the study area. Occurring in January, when nesting of terns and brown boobies is at its peak, and with very low barometric pressure (813 millibars) and a track through the northern Swains in an east-west direction, Cyclone David probably produced the most destructive wind and sea conditions and severely impacted the vegetation and birds of the Swain Reefs cays. 
Table 8 Total and vegetated areas of cays $\left(\mathrm{m}^{2}\right)$ as measured from vertical aerial photographs flown in July 1964, November 1984, June 1992, and October-November 1993, and from ground measurements in July 1983, January and July 1984, and January 1985.

$(-)=$ no data were available.

\begin{tabular}{|c|c|c|c|c|c|c|c|c|c|}
\hline \multirow{3}{*}{ Cay } & \multirow{3}{*}{$\begin{array}{l}\text { Portion } \\
\text { of cay }\end{array}$} & \multicolumn{7}{|c|}{ Area $\left(m^{2}\right)$} & \multirow{3}{*}{$\begin{array}{c}\text { Oct-Nov } \\
1993 \\
\end{array}$} \\
\hline & & July & July a & Jan. b & July b & Nov. & Jan. $^{\mathrm{b}}$ & June & \\
\hline & & 1964 & 1983 & 1984 & 1984 & 1984 & 1985 & 1992 & \\
\hline \multirow[t]{2}{*}{ Bacchi } & total & 18000 & 17200 & - & 10100 & 17200 & - & - & 19400 \\
\hline & vegetated & - & 2600 & 3500 & 1800 & 1300 & 3600 & - & 0 \\
\hline \multirow[t]{2}{*}{ Bell } & total & 46200 & 43200 & - & 44200 & 43200 & - & 33900 & 43000 \\
\hline & vegetated & 11800 & 12000 & 16300 & 13500 & 10600 & 14800 & 12700 & 14200 \\
\hline \multirow[t]{2}{*}{ Bylund } & total & 37300 & 12000 & - & - & 28400 & - & - & 27400 \\
\hline & vegetated & - & 3400 & 4400 & - & $*$ & - & - & 0 \\
\hline \multirow[t]{2}{*}{ Frigate } & total & 48100 & 42000 & - & 36200 & 42000 & - & - & 35100 \\
\hline & vegetated & 21400 & 14400 & 23600 & 19700 & 19300 & 22000 & - & 16900 \\
\hline \multirow[t]{2}{*}{ Gannet } & total & 32600 & 27200 & - & - & 27200 & - & 38100 & - \\
\hline & vegetated & 13700 & 12500 & 0 & 0 & 0 & 0 & - & 0 \\
\hline \multirow[t]{2}{*}{ Price } & total & 31600 & 28400 & - & 30400 & 28400 & - & - & 29700 \\
\hline & vegetated & - & 10800 & 16300 & 15300 & 11800 & 18500 & - & 10800 \\
\hline \multirow[t]{2}{*}{ Thomas } & total & 19900 & 16800 & - & - & 16800 & - & - & 19300 \\
\hline & vegetated & 2800 & 4000 & 0 & 0 & 0 & 0 & - & 0 \\
\hline
\end{tabular}

a Data from pedometer measurements by P. Flood.

b Data from Heatwole (1987).

* Vegetation too sparse to be visible on aerial photographs.

The total areas of the cays, and areas of vegetation, determined by either ground measurements or aerial photography for the period 1964 to 1993 are recorded in Table 8. By 1993, Bell Cay was the largest, although the vegetated area on Frigate Cay was larger. These data show that four cays lost their vegetative cover during the period of the study. Both Gannet and Thomas cays lost their vegetation between July 1983 and January 1984, Bylund during 1994, and Bacchi between January 1985 and June 1992. These dates are illustrated with a solid bar on the graphs in figures two to six.

Vegetation on Gannet Cay had been declining since about 1976 when Limpus and Lyon (1981) reported a vegetative cover of less than twenty-five percent. Prior to this time the cay had been heavily vegetated (Flood and Heatwole 1986). Remaining areas of lush vegetation were lost from the cay between December 1979 and May 1980, probably as a result of Cyclone Simon in March 1980 (Stokes 1981). By July 1980, Flood and Heatwole (1986) found "...only a few sparsely scattered plants..." remaining, and believed that the main cause of decline was 
related to the increased mobility of the cay after 1976. Three cyclones, including cyclone David, passed through the area in 1976. Gannet Cay moved some distance across the reef flat between 1964 and 1984 and changed shape from almost round to elongate (Fig. 7). In 1992, it was larger in area than previously recorded.

Thomas Cay had a similar history of vegetation loss to Gannet Cay. Flood and Heatwole (1986) reported that a decline in vegetative cover had begun by 1976, and that no plants were present by July 1980. It also moved and changed shape (Fig. 7).

The vegetation on Bylund Cay did not disappear until considerably later, but it was perhaps never as dense as that on Gannet or Thomas. Flood and Heatwole (1986) reported some vegetation still present as shoots sprouting from seed in July 1985, but no vegetation was recorded after that date. Bylund Cay perhaps changed shape least of the unvegetated cays, but still moved a considerable distance across the reef flat (Fig. 7).

Bacchi Cay appeared to almost lose its vegetation around 1979, but it subsequently recovered strongly by 1982 (Flood \& Heatwole 1986). Some vegetation was still present in January 1985. However, by 1992 no vegetation remained, and the cay had also changed its size and shape considerably (Fig. 7). 
By contrast, none of the cays which remained vegetated over the duration of the study changed in size or shape, or moved substantially (Fig. 8).

In the 100 years up to 1987, Lough (1994) reported that the interval between El Niño events varied from one to eight years. Three events were recorded in the period between 1982 and 1992 (Fig. 9a). Furthermore, the negative values of the 1982-1983 El Niño were the lowest recorded this century (Ropelewski and Jones 1987).

As might be expected, the highest monthly sea surface temperatures in the study area (Fig. 9b) generally coincided with the El Niño events reported in Figure 9a, but there were no strong trends evident in longer-term SST levels (broken line in Figure 9b). This absence of longerterm change was also evident in the data on air temperature from Gannet Cay (Fig. 9c). However, mean monthly wind speeds recorded at Gannet Cay (Fig. 9d) declined markedly during the late 1970's and mid 1980's, with a subsequent rise which leveled off around 1992.

\subsection{Patterns of human use}

The numbers of primary and secondary fishing vessels, and charter vessels sighted in each of the two relevant aerial surveillance plots (F and G) are plotted in figure 10. Smoothing splines $(l=100,000)$ were fitted to the data to assist with visual interpretation. None of the graphs in figure 10 indicated strong trends in use through time. Although both plots indicated some increase in the numbers of primary fishing vessels, these trends were produced by a very small number of data points and could not be considered reliable. The numbers of charter vessels sighted decreased in both plots after about 1991. This apparent trend was also identified by a number of long-term charter boat operators in the area (J. Hallett, M. Allen pers. comm.). 


\section{DISCUSSION}

\subsection{Study limitations}

Each island in the Swain Reefs complex has its individual history of erosion, washover and vegetation change and these may be reflected in different responses of their bird populations. Other factors will most likely have influenced each island and their seabird populations equally. This section attempts to interpret observed fluctuations in these populations in the light of known environmental events.

There were a number of limitations associated with the study, and these must be kept in mind. Not all seabird breeding islands in the Swain Reefs could be visited each year. Bell Cay lies outside of the Swain complex proper and is more exposed to the effects of bad weather. It is also remote from the other cays and could not be visited on every trip. Seabirds also breed on Riptide and Distant Cays which lie further to the north, but their remote location prevented regular access. On some occasions, especially on Bell Cay, birds were censused but nests were not, because of concerns about disturbance in the presence of large numbers of predatory gulls.

A population of seabirds on an island may contain non-breeding individuals in addition to breeding pairs. For this reason, neither counts of nests alone nor counts of adults alone may give a true picture of the total resident population. The breeding population can be reliably assumed to be at least twice the number of nests counted; however, large numbers of adult birds of many species were also observed on the beaches of the Swain Reefs cays. Some of these individuals may have been breeding adults taking a rest from their nesting duties, but most were likely to have been unattached, non-breeding adults. Nevertheless, an unknown and varying proportion of the total population would also have been feeding at sea at the times the counts were taken. The proportion present would be influenced by factors such as the stage of breeding, the time of day, the weather conditions and the availability of food. The size of total resident populations of each species (breeding plus non-breeding adults) were therefore difficult to determine accurately.

Jones et al. (in review) have shown that the numbers of seabirds of various species changes during the diel cycle on the Swain Reefs cays, with largest differences observed between day 
and night. In the present study, only daytime counts were included. However, while it was intended to census every island each year and at equivalent months and times of day, this ideal was not always attained. The summer expeditions did not always take place during the same month because of obligations of the investigators during that time, and occasionally because of inclement weather. These censuses spanned the months of November to February and were potentially more variable due to seasonal differences than the winter counts, all of which were carried out in July. However, if the counts made in any particular summer month are examined in isolation (Figs. 2 to 6), apparent trends do not change on most occasions, possibly indicating that count variation from this source was minimal. The censuses were not always conducted at precisely the same time of day, even though most counts were completed during the morning before 0900 hours. Seasonal and diurnal variation in bird's foraging schedules may therefore have contributed to some variability in the data.

Analysing combined counts of both nests and adult birds across the various cays reduced the effects of island-specific biases, such as environmental factors, which may have produced significant within-island population trends. These "TOTALS" graphs (Figs. 2 to 6) contain fewer data points than the graphs for individual cays, because inclement weather conditions or the presence of predatory gulls at times precluded counting (particularly nests) on some cays. When this occurred, data from that particular expedition were omitted. In total, data from 11 summer and 10 winter expeditions were included. The data in these graphs should be viewed first during discussion of population trends.

\subsection{Seabird populations}

With the available information, it was difficult to determine the effects that various cyclones and other periods of severe weather had on the seabirds, vegetation and cay morphology in the Swain Reefs. Four cays lost their vegetation during the period of the study, but these losses cannot be fully explained by cyclonic effects, although this was probably a key element. Island wash-over and erosion during cyclones, disturbance by turtles and seabirds, extended periods of drought, and human disturbance (Gannet Cay) were possibly all contributing factors to vegetation removal (Heatwole 1987). Vegetation was an important morphological stabilising factor on islands, as indicated by the relatively small changes in shape and size of the vegetated cays compared to the unvegetated ones (compare Figs. 7 and 8). Vegetative cover was also a prerequisite for breeding for some species of seabird such as the least frigatebird, 
common noddy, bridled tern and sooty tern, and those species disappeared from cays that lost their vegetation.

The populations of some species of seabirds in the Swain Reefs appears to be stable. However, it was not possible to always accurately count all species. The smaller groundnesting species that breed amongst or under the low vegetation on some cays presented counting difficulties at time of nesting. Bridled terns (Sterna anaethetus), sooty terns (Sterna fuscata), and common noddies (Anous stolidus) belong to this category. Crested terns (Sterna bergii), lesser crested terns (Sterna bengalensis), and black-naped terns (Sterna sumatrana) could be counted relatively easily but nested sporadically, and lesser frigatebirds (Fregata ariel) nested on only one island, Bell Cay. However, although these species were excluded from the trend analyses because of unsuitable data, there was no indication that their populations changed during the period of the study.

The brown booby (Sula leucogaster), the masked booby (Sula dactylatra), and the silver gull (Larus novaehollandiae), were therefore the only species analysed for trends in population size and numbers of nests, although silver gull nesting was not analysed because the nests of gulls are well camouflaged, and few were located. Discussion will concentrate on these three species.

The time of breeding and the degree of breeding synchronisation of the brown booby is highly variable, both between and within colonies in different parts of the world (Nelson 1978; Harrison 1990). Gill (1994) reported that nesting on Ascension Island occurs at eight-month intervals. The time of nesting of the masked booby is also highly variable around the world, but it is primarily a summer nester in the Western Pacific (Nelson 1978). This variability of timing of breeding, together with the possibility of double nesting seasons (Serventy et al. 1971), and the large seasonal variation in nesting numbers (Figs. 2, 3), increases the difficulty of interpreting bi-annual counts for either booby species in the Swain Reefs. However, the sulids have a protracted breeding cycle. Nelson (1978) reported that the breeding cycles (including the prelaying period) of the brown and masked booby to be 214 to 223 days, and 159 to 164 days, respectively. Counts at six-month intervals, therefore, should have recorded all nesting masked boobies and most nesting brown boobies at some stage of their breeding cycles. 
As reported in the results, the brown booby is predominantly a summer nester in the Swain Reefs. The sympatric masked booby, in contrast to both its reported nesting season in the region and to the brown booby, tended to nest in winter in the Swain Reefs (Fig. 3). On most cays, the mean number of masked booby nests counted in winter was significantly higher than in summer, whereas the numbers of adults counted was not significantly different between seasons (Table 5). These data possibly indicate that most of the breeding-age masked boobies remain in the area throughout the year, which contrasts with the 'migratory or dispersive' behaviour generally reported for the species (Marchant \& Higgins 1992). To help resolve this uncertainty an extensive mark-recapture study was completed (O'Neill et al. in review).

Suitable nesting habitat is probably a significant limiting factor to the size of breeding populations for a number of seabird species in the Swain Reefs. Tree-nesting species are, of course, absent from the area, and species requiring vegetative ground cover for nesting, such as the common noddy and bridled tern, are limited to the three vegetated cays (four including Distant Cay).

Nelson (1978) reported that nesting density in both the brown and masked booby is highly variable. He quoted an example for the brown booby of one nest every 13.4 square metres, and an optimal density for the masked booby of 201 square metres per nest, although densities of up to one nest every 3.3 square metres were reported. Nest densities of boobies on the Swain cays (Table 6) were comparatively low, except for the densities of masked boobies on Gannet and Price Cays, which were close to the optimal density suggested by Nelson (1978). Gannet Cay is not now vegetated, and recorded both the highest numbers and nesting densities of masked boobies. The other non-vegetated cays (Bacchi, Bylund and Thomas) are considerably smaller (Table 8) and subject to more regular overwash during severe weather (pers. obs.), reducing their suitability for nesting. However, nesting densities for neither species approached the limits reported by Nelson (1978), even on the more stable cays. Factors other than availability of suitable breeding habitat must, therefore, have limited the population size of the masked booby in the Swain Reefs, and produced the reported declines in the brown booby.

Nelson (1978) found that the food supply of the masked booby is so irregular that complete breeding failure can occur. Even in comparatively good times, the inability of parents to sychronise their return to the chick with food often brought them to the brink of starvation. 
The resulting relatively long time to fledging further increases the risks of chick mortality by other means. These environmental and life history factors are probably the dominant population limiting mechanisms in the Swain Reefs.

The brown booby incurred substantial declines during the study period, both in the number of nests and adult birds counted. Negative population trends were observed on all the islands, except Bell Cay, where fewer census points made interpretation difficult. The summer regression lines in the "TOTALS" graphs (Figs. $2 \& 4$ ) illustrate the cumulative declines that occurred on individual cays. They indicate that the trend occurred generally throughout the area and was not simply a migration pattern from some islands to others. The causes of this significant decline are unclear; however, they are unlikely to be human-induced. The aerial surveillance data presented in figure 10 do not suggest recent increases in the level of human visitation to the area. There are no data to suggest recent change in charter or fishing operations that could have caused higher levels of seabird disturbance.

Direct competition between seabirds and fisheries has the potential to seriously impact seabird populations (e.g. Jones \& DeGrange 1988). The brown booby is known to feed on various species of small, shallow water reef fishes in other parts of the world (Harrison 1990), and is known to be highly opportunistic in its feeding habits (Harrison et al. 1984). On the Great Barrier Reef, the brown booby feeds on a wider variety of prey than the masked booby (Blaber et al 1995). Preliminary examination of regurgitate samples collected from the Swain Reefs generally confirms this varied dietary composition (Blaber, pers. comm.). The masked booby might therefore be expected to find it most difficult to feed during times of reduced fish availability. Because the brown booby is also known to take trawl fishery discard (Blaber et al. 1995), increases in fishing activity would possibly favour its survival.

All the seabird breeding islands in the Swain Reefs are surrounded by marine park zoning, which prohibits fishing. The brown booby feeds largely inshore (Nelson 1978), but the distance the species travels from its breeding colonies in this area to feed is not known. While the reefs immediately surrounding the breeding colonies are protected from fishing, those a little more distant are not, and it is possible that loss of food from those reefs could impact the ability of adult brown boobies to successfully feed and rear young. However, the waters surrounding the large colonies of brown boobies breeding on Fairfax and Hoskyn islands further to the south, are subject to similar but more intensive fishing pressures. Those colonies do not appear 
to have declined, and although no data are available to confirm this, it seems unlikely that depletion of commercial fish species in the Swain Reefs would be sufficient to cause the observed declines in brown booby numbers. Furthermore, there was no earlier peak in fishing effort in the Swain Reefs (Gribble, pers. comm.) which may have artificially inflated the brown booby population via the provision of fishery discard.

Physical changes to the nesting habitat of the brown booby were pronounced in the Swain Reefs. Four of the seven nesting islands lost their vegetative cover during the study period and two, Bylund and Frigate, had reductions in total area of about 27 percent. Brown boobies preferentially nest in low vegetation and will use it for shelter (Marchant and Higgins 1992; Frith 1983; Pizzey 1982). Presumably loss of vegetation would affect breeding success. However, similar declines in numbers of adults and nests occurred both on the stable islands and on the islands that lost their vegetation and/or were reduced in size. Loss of vegetation or reduction in size of cays were, therefore, unlikely to be the primary causes of the observed population decline in the brown booby.

A major source of short-term climatic variation in the Great Barrier Reef region are the extreme values of the El Niño Southern Oscillation (ENSO) (Lough 1994). Oceanographic conditions which affect food supply have been identified as important factors in the determination of breeding success in seabirds, including booby species (e.g. Reville \& Stokes 1994; Shreiber \& Schreiber 1984). In particular, warm water is known to have negative effects on seabird populations by decreasing the availability of prey species (Wilson 1991), but periods of poor weather may also produce sea surface conditions less suitable for the capture of prey by some (particularly smaller) seabird species (Hulsman 1988). The data presented in figure 9a show that four significant climatic events associated with the ENSO occurred during the study period, and resulted in periodic elevations in sea surface temperatures. However, mean monthly sea surface temperatures showed no long term change in Great Barrier Reef waters during this period (Fig. 9b).

The occurrence of high numbers of emaciated brown booby chicks found during capture for banding, further suggests that food availability played a major role in the observed decline of the species. It is possible that repeated abnormal temperature elevations and associated depletions of prey species, produced successive failures of the brown booby breeding effort over the past decade or so. The periodicity of these El Niño events was possibly shorter than 
the recovery time required by the booby population. This is likely to have reduced the mean age and level of experience of the breeding population, and further reduced its ability to recover (see Ainley et al 1984).

The masked booby may not have exhibited similar declines to the brown booby because of differences in its feeding ecology. Even though it is a less opportunistic feeder than the brown booby, it tends to feed further from land in more oceanic waters (Nelson 1978), and its food source may not have been as severely affected.

The succession of El Niño events after the severe one of 1982-1983 was possibly a compounding problem for the brown booby (and other summer-nesting seabirds of the area), that produced declines in population size. It is suggested, therefore, that population change (for the brown booby at least) may be part of a longer term cyclic process. Only a continuation of the current population monitoring program will test this hypothesis. This is desirable, as Harrison (1990) suggested that brown booby populations in many areas of the world are small and threatened. This is despite the species generally being considered the most common of the sulids, with a widespread distribution throughout tropical and subtropical oceans (Nelson 1978).

The silver gull was the only other species observed to decline in numbers during the period of the study. This species nests at any time of the year on the Great Barrier Reef, but with a summer preference in the southern reef (Smith 1992). In other parts of Australia, the timing of breeding is extremely variable, but perhaps with a slight winter preference (see for example Wheeler \& Watson 1963; Pringle 1987; Ottaway et al. 1988). The clear preference of the silver gull to nest in winter in the Swain Reefs confounds any hypothesis that its observed decline is directly connected to the decline in the brown booby, one of its principal kleptoparasitic targets but primarily a summer nester. Spilled or stolen regurgitate from booby parents attempting to feed their chicks is an important part of the silver gull's food source, but its availability cannot be considered critical for silver gull breeding. Significant silver gull declines only occurred on the cays that lost their vegetation (Fig. 6, Table 3), which is not surprising given the known preference of the species to nests on islands where short vegetation provides their chicks with adequate cover (Pringle 1987). While the observed decline in the brown booby may have decreased the availability of food for the silver gull, the primary cause of the gull decline was apparently the loss of vegetation from Bacchi, Bylund, Gannet and Thomas Cays. Because the population size remained comparable between 
summer and winter, and declined significantly in both seasons, the rates of immigration and emigration from this population are probably low.

The decline in the silver gull possibly also indicates that current levels of human visitation to the cays are minimal, as gull population declines in Australia are a rare occurrence, with numbers usually burgeoning in response to human development or visitation in coastal areas (Smith 1992). Perhaps silver gulls from the Swain Reefs are moving to adjacent coastal areas such as Yeppoon and Mackay for more assured food resources. Nevertheless, there has been marked behavioural change in the silver gulls of the Swain Reefs over the past ten years, with kleptoparasitic activity now stronger in association with investigator presence. This apparent increase in aggressive behaviour toward other nesting seabirds, particularly predation of eggs, led to an early belief that the numbers of silver gulls in the area was increasing, but the data clearly shows this not to be the case. The impact of increased kleptoparasitic behaviour on the breeding populations of other seabirds is unknown, but it is expected to be minimal due to the relatively small and decreasing numbers of silver gulls present.

\subsection{Management of seabird islands in the Swain Reefs}

None of the seabirds observed breeding in the Swain Reefs appear on lists of Australian rare and threatened species. However, little is known of their population biology, and their isolation from other populations may increase their conservation importance. Until shown to be otherwise, the seabird populations in the Swain Reefs should be treated as geographic isolates and managed accordingly.

It has been argued elsewhere (e.g. Drury 1979; Craig 1994) that one of the best defences a population has against extinction is the existence of a meta-population, where a number of subpopulations exist which allow some, but not too much, gene flow between them. Drury (1979) also emphasised that when managing seabird islands it is most important to protect the colonies that are reproductively successful and exporting young, and to maintain a variety of sites so that populations may move as local patterns of prey productivity shift. In particular, it is important to protect those islands where seabird populations survive during hard times, when feeding is difficult. These refugia (see Andrewartha \& Birch 1984) allow recolonisation of the local area when conditions improve. The distribution of the colonies in the Swain Reefs area, and their relatively undisturbed status, allow these principles to be adopted with relative ease. 
Not all of the nine coral cays in the Swain Reefs currently used by seabirds for breeding are of equal importance to seabird conservation. The three larger, vegetated cays (Bell, Frigate and Price) support substantially more breeding species (nine, ten and seven respectively) than the others (Table 1), and larger total numbers of breeding seabirds (Table 2). Gannet Cay remains locally important for the booby species (Table 2). Given the observed declines in some species, particularly the brown booby, it is essential these four cays at least are safeguarded from possible negative, and little understood, effects of human visitation. They contain a suite of breeding species representative of those occurring on the Great Barrier Reef, and therefore remain useful as baseline populations for comparison with areas more affected by expanding tourist and recreational pressures. Extensive data from a long-term mark and recapture program (O'Neill et al. in review) indicate that these four cays are also the most productive for the two booby species.

All cays in the Swain Reefs are relatively small, and even brief forays ashore by unknowing visitors can cause considerable disruption to breeding, especially during sensitive stages of the nesting cycle. However, access to some cays is considered an important benefit for tourism by charter vessel operators. Because of the variability in timing of seabird breeding, both within and between species, it is not possible to close all islands effectively for only part of the year and protect breeding seabirds. The only safe management measure is total closure of the four most important seabird breeding cays (Bell, Frigate, Gannet and Price). The remaining cays, which are scattered throughout the area and therefore amenable to users, should remain open for visitation, but with access limited to below the high water mark. A monitoring scheme that will accurately determine the frequency and duration of human visitation to the cays is also required, to verify the assumption of negligible human impact on breeding seabirds.

\section{CONCLUSION}

These results, representing 12 years of bi-annual seabird censuses in a remote part of the Great Barrier Reef, show that the breeding populations of most of the seabirds in the Swain Reefs appear to be stable and secure. However, the brown booby has undergone a marked decline in both the number of breeding pairs and the number of adults present. The silver gull has declined by a similar magnitude. A number of more cryptic species such as the black- 
naped tern, bridled tern and common noddy, may also have declined, but difficulties with censusing precluded a reliable appraisal.

A major reason for the declines may have been a reduction in available food supply associated with elevated sea surface temperatures induced by El Niño. There is some evidence to suggest that the declines may not be permanent, but part of long term population fluctuations. A continuation of monitoring is needed to test this hypothesis.

The Swain Reefs support a small but varied avifauna, with the breeding composition different from any other in the southern Great Barrier Reef. The islands considered most important to the long term viability of seabird populations in the area have been identified, and an appropriate management strategy suggested. From a management perspective, the area is unique in that biological baseline information was recorded before major human impact may have occurred. Remoteness has been an advantage, creating an opportunity to make informed management decisions prior to the establishment of significant and undesirable patterns of visitation.

\section{ACKNOWLEDGMENTS}

We are grateful to the many students, volunteer helpers and staff from the Queensland Department of Environment that assisted in the collection of data over the years. They are too numerous to all be acknowledged individually but Peter Saenger, Felicity Savage, Petah Abbott, Sheryl Greathead, Bruce Knuckey and the late Terry Walker merit special mention. Earlier manuscripts were greatly improved by critical review from Kees Hulsman, Tony Stokes, Emma Gyuris, and Don Cook. Assistance with statistics came from Glenn De'ath and Alan Lyle. Lyn O'Neill produced the maps. The field work was supported by funds from the Australian Research Grants Scheme, Marine Science and Technology, Internal Research Funds of the University of New England, the North Carolina Agricultural Research Service, and the Queensland Department of Environment. The Cooperative Research Centre (Reef Research Centre) and the Queensland Department of Environment provided funds for the compilation and analysis of the data, and completion of the report. 


\section{REFERENCES}

Ainley, D.G., Carter, H.R., Anderson, D.W., Briggs, K.T., Coulter, M.C., Cruz, F., Cruz, J.B., Valle, C.A., Fefer, S.I., Hatch, S.A., Schreiber, E.A., Schreiber, R.W., \& N.G. Smith. 1984. Effects of the 1982-83 El Niño - Southern Oscillation on Pacific Ocean Bird Populations. Symposium 29. Birds and the El Niño Southern Oscillation. Eds. R.W. Schreiber and D.C. Duffy.

Andrewartha, H.G. \& L.C. Birch. 1984. The Ecological Web. More on the Distribution and Abundance of Animals. The University of Chicago Press, Chicago.

Blaber, S.J.M., Milton, D.A., Smith, G.C., \& M.J. Farmer. 1995. Trawl discards in the diets of tropical seabirds of the northern Great Barrier Reef, Australia. Marine Ecology Progress Series 127, 1-13.

Costello, J.W. 1978. Birds in the Swain Reefs, Queensland. The Australian Bird Watcher June, 1978: 205-208.

Craig, J.L. 1994. Meta-populations: is management as flexible as nature? In Olney, P.J.S., Mace, G. M. \& A.T.C. Feistner. Creative Conservation: Interactive management of wild and captive animals. Chapman \& Hall, London.

Drury, W.H. 1979. Population Dynamics in Northern Marine Birds. In Bartonek, J.C. \& D.N. Nettleship. Conservation of Marine Birds of Northern North America. Wildlife Research Report 11, U.S. Dept. of the Interior, Fish \& Wildlife Service. Washington, D.C.

Flood, P.G. \& H. Heatwole. 1986. Coral cay instability and species-turnover of plants at Swain Reefs, southern Great Barrier Reef, Australia. J. Coastal Research 2, 479-496.

Frith, H.J. (ed.). 1983. Reader's Digest Complete Book of Australian Birds. Reader's Digest Services Pty. Ltd. Sydney.

Gill, F.B. 1994. Ornithology. W.H. Freeman and Company, New York.

Gillett, K. \& F. McNeill. 1959. The Great Barrier Reef and adjacent isles. Coral Press, Sydney.

Harrison, C.S. 1990. Seabirds of Hawaii; Natural History and Conservation. Cornell Univ. Press, Ithaca.

Harrison, C.S., Hida, T.S. \& M.P. Seki. 1984. The diet of the brown booby Sula leucogaster and Masked Booby Sula dactylatra on Rose Atoll, Samoa. Ibis 126, 588-590.

Hastie, T.J. \& R.J. Tibshirani. 1990. Generalized Additive Models. Chapman \& Hall, London.

Heatwole, H. 1981. A Coral Island. The Story of One Tree Reef. Collins, Sydney. 
Heatwole, H. 1987. Some aspects of phytogeography and Vegetation Dynamics of Islands of the Great Barrier Reef. Ph.D. (Botany) Thesis, University of Queensland, 367pp.

Heatwole, H. 1991. Factors affecting the number of species of plants on islands of the Great Barrier Reef, Australia. Journal of Biogeography 18, 213-221.

Heatwole, H. \& T.A. Walker. 1989. Dispersal of Alien Plants to Coral Cays. Ecology 70(3), 787-790.

Hindwood, K.A. 1961. Coral Sea Trip. Coral Sea trip, November 1961 aboard the H.M.A.S. Gascoyne. Unpublished field notes.

Hulsman, K. 1988. The Structure of Seabird Communities: Australia. In Joanna Burger (ed.). Seabirds and Other Marine Vertebrates; Competition, Predation and Other Interactions. pp. 59-91. Columbia Univ. Press, New York.

Jones, L.L. \& A.R. Degrange. 1988. Interactions Between Seabirds and Fisheries in the North Pacific. In Burger, J. (ed.). Seabirds and Other Marine Vertebrates; Competition, Predation and other Interactions. Columbia University Press, New York.

Jones, M., Heatwole, H. \& J.P. O'Neill. Diel changes in numbers of seabirds occupying cays on the Swain Reefs, Great Barrier Reef, Australia. Submitted to Marine Ornithology.

Lane, S.G. \& H. Heatwole. 1991. Seabird Islands No. 214. Distant Cay, Great Barrier Reef, Queensland. Corella 15, 117-118.

Limpus, C.L. \& B.J. Lyon. 1981. Seabirds Breeding on the Swain Reefs, Queensland. Corella 5(5), 101-105.

Lindsey, T.R. 1986. The Seabirds of Australia. The National Photographic Index of Australian Wildlife. Angus \& Robertson Publishers.

Lough, J.M. 1994. Climate variation and El Niño-Southern Oscillation events on the Great Barrier reef: 1958 to 1987. Coral Reefs 13, 181-195.

Marchant, S. \& P.J. Higgins. 1990. Handbook of Australian, New Zealand \& Antarctic Birds. Volume 1, Part B, Australian Pelican to Ducks. Oxford University Press, Melbourne.

McMichael, D.F. 1963. The Swain Reefs Expedition. Australian Natural History September, $1963,210-214$.

Moverley, J.H. 1985. Winter bird observations for cays in the southern section of the Swain Reefs, Queensland. The Sunbird 15 (4), 43-79.

Nelson, J.B. 1978. The Sulidae; Gannets and Boobies. Oxford University Press.

O’Neill, P. \& H. Heatwole. 1996. Riptide Cay, Great Barrier Reef, Queensland. Corella 20(3), 11-112. 
O'Neill, P., Heatwole, H., Preker, M. \& M. Jones. In review. Populations, Movements and Site Fidelity of Masked and Brown Boobies on the Swain Reefs, Great Barrier Reef, as shown by Banding Recoveries.

Ottaway, J.R., Carrick, R. \& M.D. Murray. 1988. Reproductive Ecology of Silver Gulls, Larus novaehollandiae Stephens, in South Australia. Aust. Wildl. Res. 15, 541-56.

Pizzey, G. 1982. A Field Guide to the Birds of Australia. Collins, Sydney.

Pringle, J.D. 1987. The Shorebirds of Australia. Angus and Robertson, Sydney.

Reville, B.J. \& T. Stokes. 1994. Conservation of seabirds on Christmas Island, Indian Ocean. In Nettleship, D.N., Burger, J., and M. Gochfield. Seabirds on Islands; Threats, Case Studies and Action Plans. pp. 244-257. Birdlife International, Cambridge.

Ropelewski, C.F. \& P.D. Jones. 1987. An extension of the Tahiti-Darwin Southern Oscillation Index. Monthly Weather Rev. 115, 2161-2165.

Serventy, D.L., Serventy, V.N. \& J. Warham. 1971. The Handbook of Australian Seabirds. A.H. \& A.W. Reed Ltd, Sydney.

Schreiber, R.W. \& E.A. Schreiber. 1984. Central Pacific seabirds and the El Niño Southern Oscillation: 1982 to 1983 perspective. Science 225, 713-716.

Smith, G.C. 1992. Silver gulls and emerging problems from increasing abundance. Corella $16(2), 39-46$.

Smith, J.M.B., Heatwole, H., Jones, M. \& B.M. Waterhouse. 1990. Drift disseminules on cays of the Swain Reefs, Great Barrier Reef, Australia. Journal of Biogeography 17, 517.

Stokes, T. 1981. Coral Sea Islands Nature Conservation Program, Report of the Fourth Biological Survey and Monitoring Visit - 15 to 27 May 1981. Internal Report, ANCA.

Stokes, T., Hulsman, K., Ogilvie, P. \& P. O'Neill. 1996. Management of Human Visitation to Seabird Islands of the Great Barrier Reef Marine Park Region. Corella 20(1), 1-13.

Walker, T.A. \& M.E. Jones. 1986a. Seabird Islands No. 164. Frigate Cay, Great Barrier Reef, Queensland. Corella 10, 89-90.

Walker, T.A. \& M.E. Jones. 1986b. Seabird Islands No. 165. Bylund Cay, Great Barrier Reef, Queensland. Corella 10, 91-92.

Walker, T.A. \& M.E. Jones. 1986c. Seabird Islands No. 166. Price Cay, Great Barrier Reef, Queensland. Corella 10, 93-94.

Walker, T.A. \& M.E. Jones. 1986d. Seabird Islands No. 167. Bell Cay, Great Barrier Reef, Queensland. Corella 10, 95-97. 
Walker, T.A. \& M.E. Jones. 1986e. Seabird Islands No. 168. Gannet Cay, Great Barrier Reef, Queensland. Corella 10, 98-100.

Walker, T.A., Jones, M.E. \& F. Savage. 1989. Seabird Islands No. 195. Thomas Cay, Great Barrier Reef, Queensland. Corella 13, 51-52.

Wheeler, W. R. \& I. Watson. 1963. The Silver Gull. Ети 63(2), 99-173.

Wilson, U.W. 1991. Responses of three seabird species to El Niño events and other warm episodes on the Washington coast, 1979-1990. The Condor 93, 853-858. 
Appendix 1 Record of ornithological expeditions to the Swain Reef's cays.

\begin{tabular}{|c|c|c|c|c|c|c|c|c|c|c|c|c|c|c|}
\hline \multirow[t]{2}{*}{ Date } & \multicolumn{13}{|c|}{ Cay } & \multirow[t]{2}{*}{ Recorder } \\
\hline & $\mathrm{Bac}$ & Byl & Bel & Dis & $\mathrm{Fri}$ & Gan & Hor & Hix & Lav & Pri & Tho & $\begin{array}{c}\mathrm{Zo} \\
\mathrm{d}\end{array}$ & Rip & \\
\hline Oct 60 & $\bullet$ & $\bullet$ & & & $\bullet$ & 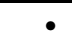 & & $\bullet$ & & $\bullet$ & $\bullet$ & & & Thomas \\
\hline Jan 62 & & & & & • & & & & & & & & & Mc Michael \\
\hline Aug 67 & & & & & & & & & & & & • & & Heatwole \\
\hline Nov 67 & & & & & & - & & & & & & & & Heatwole \\
\hline Jul 69 & & & & & • & & & & & & & & & Costello \\
\hline Jun 71 & & & & & & & & • & & & & & & Unknown \\
\hline Nov 76 & • & • & - & & • & - & & & $\bullet$ & • & - & & - & Limpus \& Lyon* \\
\hline Apr 77 & & & & & - & & & & & & & & - & Costello \\
\hline Jul 79 & • & & & & • & • & & & & & & & & Heatwole \\
\hline $\operatorname{Dec} 79$ & & & & & & - & & & & & & & & ANPWS \\
\hline May 80 & & & & & & $\bullet$ & & & & & & & & ANPWS \\
\hline May 81 & & & & & & - & & & & & & & & Veenstra \\
\hline Jul 81 & & & $\bullet$ & & & & & & & & & & & Heatwole \\
\hline Dec 81 & & & & & & • & & & & & & & & ANPWS \\
\hline Jul 82 & • & • & - & & • & - & & & & • & - & & & Heatwole* \\
\hline Nov 82 & & & & & & - & & & & & & & & ANPWS \\
\hline Jul 83 & • & • & $\bullet$ & & - & - & & - & & - & - & & & Heatwole, Moverley * \\
\hline Jan 84 & • & • & & & • & $\bullet$ & & & & • & $\bullet$ & & & Reid* \\
\hline Jul 84 & • & • & & & • & $\bullet$ & & & & • & $\bullet$ & & & Moverley \\
\hline Jan 85 & $\bullet$ & • & • & & - & • & & & & - & $\bullet$ & & - & Heatwole, Jones* \\
\hline Jul 85 & • & • & $\bullet$ & & • & - & & & & • & - & & & Heatwole, Jones* \\
\hline Feb 86 & • & - & - & & • & - & - & & - & • & $\bullet$ & & & Heatwole, Jones* \\
\hline Apr 86 & • & - & & & - & - & & & & - & $\bullet$ & & & Walker \\
\hline Jul 86 & • & - & - & & • & - & & & & - & - & & & Heatwole, Jones* \\
\hline Jan 87 & - & • & & & - & - & - & & & - & - & & & Heatwole, Jones* \\
\hline Jul 87 & • & - & • & & • & - & & & & $\bullet$ & - & & & Heatwole, Jones* \\
\hline Jan 88 & - & - & $\bullet$ & & • & - & - & & & - & - & & & Heatwole, Jones* \\
\hline Jul 88 & - & • & $\bullet$ & & - & $\bullet$ & & & & $\bullet$ & $\bullet$ & & & Heatwole, Jones* \\
\hline Nov 88 & - & - & & & • & - & & • & & - & - & & & Domm* \\
\hline Jan 89 & - & - & & - & • & - & & & - & $\bullet$ & • & • & & Heatwole* \\
\hline Apr 89 & - & $\bullet$ & & - & • & - & & & & $\bullet$ & • & & - & Lee \\
\hline Jul 89 & - & • & & & • & - & & & & $\bullet$ & $\bullet$ & & & Heatwole, Lane* \\
\hline Nov 89 & & & & & & $\bullet$ & & & & & & & & Prythech \\
\hline Nov 89 & $\bullet$ & $\bullet$ & & & • & • & & & & • & • & & & Upton * \\
\hline Jan 90 & • & • & & & • & $\bullet$ & $\bullet$ & & & $\bullet$ & - & & & Heatwole* \\
\hline Sep 90 & & & & & & • & & & & & & & & Walker \\
\hline Jul 91 & • & • & $\bullet$ & & - & $\bullet$ & & & & $\bullet$ & $\bullet$ & & & Heatwole* \\
\hline Nov 91 & - & - & & & • & $\bullet$ & $\bullet$ & - & & $\bullet$ & $\bullet$ & & & O'Neill* \\
\hline Jul 92 & - & - & • & & • & - & & & & $\bullet$ & $\bullet$ & & & Heatwole, Abbott* \\
\hline Oct 92 & & & - & & & & & & & & & & & Knuckey \\
\hline Nov 92 & - & - & & & • & - & & & & $\bullet$ & • & & & O'Neill, Ogilvie* \\
\hline May 93 & & & & & • & - & & & & & & & & O'Neill \\
\hline Jul 93 & - & - & - & & • & - & & $\bullet$ & & - & $\bullet$ & & & Heatwole, O'Neill* \\
\hline Dec 93 & - & • & - & & - & - & & & & - & - & & & O'Neill* \\
\hline Mar 94 & & & - & & & & & & & & & & & Knuckey \\
\hline Mar 94 & • & • & & & - & - & & & & - & - & & & O'Neill \\
\hline Jul 94 & $\bullet$ & $\bullet$ & $\bullet$ & & $\bullet$ & $\bullet$ & & $\bullet$ & & $\bullet$ & $\bullet$ & & & Heatwole* \\
\hline
\end{tabular}

* Signifies expeditions from which seabird counts were used for trend analyses.

Bac=Bacchi Cay, Bel=Bell Cay, Byl=Bylund Cay, Dis=Distant Cay, Fri=Frigate Cay, Gan=Gannet Cay, Hix=Hixson Cay, Hor=Horsehoe Reef (wreck), Lav=Lavers Cay, Pri=Price Cay, Rip=Riptide Cay,

Tho=Thomas Cay, Zod=Zodiac Cay. 
